\title{
HIGHER-ORDER FEATURE-PRESERVING GEOMETRIC REGULARIZATION
}

\author{
MARC DROSKE* AND ANDREA BERTOZZI ${ }^{\dagger}$
}

\begin{abstract}
We introduce two fourth-order regularization methods that remove geometric noise without destroying significant geometric features. These methods leverage ideas from image denoising and simplification of high contrast images in which piecewise affine functions are preserved up to infinitesimally small transition zones. We combine the regularization techniques with active contour models and apply them to segmentation of polygonal objects in aerial images. To avoid loss of features during the computation of the external driving forces we use total-variation based inverse scale-space techniques on the input data. Furthermore, we use the models for feature-preserving removal of geometric texture on surfaces.
\end{abstract}

1. Introduction. Geometric partial differential equations are very powerful and widely used ingredients for modeling and solving problems that involve "shapes" as free variables [10, 34, 33]. Such PDEs can be modeled directly or emerge from variational methods by either deriving the formal Euler-Lagrange equations or the correspondig gradient descent (see [50, 17, 51]). One prominent example is the problem of image segmentation, which is often modeled in terms of the boundary of the unknown segment. In particular, the well-known class of active contour models [14, 23, 49, 13, 38, model a geometric evolution problem, that evolves an initial curve towards the boundary of the segment. Naturally, the main component that influences the segmentation process is the external data, which can be images of any kind, such as photographs or magnetic resonance images. Since images can contain a very high amount of information and in addition be corrupted by noise, it is often crucial to mitigate non-uniqueness by imposing a restriction on the complexity of the solution. This reduction of the solution space by penalization is referred to as regularization and is often achieved by adding smoothness to the evolving geometry. In this context, a common problem is to find a good balance between the smoothing process and external driving forces. Our aim in this paper is to exploit prior knowledge to devise feature-preserving smoothing methods, that help to avoid oversmoothing at sharp corners. In particular, these methods facilitate the segmentation of objects with only a piecewise smooth boundary by avoiding over-smoothing.

Let us first describe some of the most important classic approaches for boundarybased segmentation. Consider, for example, the pioneering work of Kass, Witkin and Terzopoulos [37. which introduced active contours 14. (also known as snakes) as parametrized curves or surfaces that evolve according to both local properties of the curve and to image-dependent forces that are directed towards significant features. The classical image snake method computes minimizers of the functional

$$
E[c]=\frac{\alpha}{2} \int_{0}^{1}\left\|c_{z}(z)\right\|^{2} \mathrm{~d} z+\frac{\beta}{2} \int_{0}^{1}\left\|c_{z z}(z)\right\|^{2} \mathrm{~d} z-\frac{1}{2} \int_{0}^{1}\left\|\nabla u_{0}(c(z))\right\|^{2} \mathrm{~d} z
$$

to segment boundaries for the input image $u_{0}: \Omega \rightarrow \mathbb{R}$ on the image domain $\Omega \subset$ $\mathbb{R}^{d}$. The functional $E$ is defined on closed curves given by a parametrization $c$ : $[0,1] \rightarrow \Omega$ and $\alpha, \beta$ are positive weights of the respective energy contributions. The

\footnotetext{
${ }^{*}$ Department of Mathematics, University of California. Current affiliation: mental images, Fasanenstr. 81, 10623 Berlin, Germany marc@mental.com

${ }^{\dagger}$ Department of Mathematics, University of California, 405 Hilgard Avenue Los Angeles, CA 90095-1555, USA bertozzi@math.ucla.edu
} 
first two, the membrane energy and the thin-plate energy, control the fairness of the curve and constitute the internal energy. The last integral defines the external energy, i. e., models the image feature driven dependence of the curve: the lower the gradient of $u_{0}$ is in magnitude, the stronger is the penalization of the curve. In a gradient-flow minimization method, the external energy is responsible for evolving the curve so that it eventually stops at a boundary, whereas the internal energy smoothes rough edges. However, if the internal energy (regularization) does not reflect our expectations on the shape of the object (e.g., segmentation of smooth shapes versus polygons or objects with a fractal or fuzzy boundary), the balancing of the energies is a difficult trade-off. Ideally, the regularization energy should only penalize implausible shapes. Naturally, plausibility depends highly on the context. In medical image segmentation, a smoothness assumption is perfectly valid, in this paper however we would like to devise a regularization energy, which treats "piecewise" smooth shapes, such as smooth approximations to polygons as plausible.

Active contour models, including all their variants, are commonly used in the literature and successfully applied to a wide range of segmentation problems, especially in medical imaging (cf. e.g. [57, 18]). Even though parametric models have serious difficulties in changing their topology, they are numerically appealing because they can be solved very efficiently. In many applications the topology is known a-priori and hence no splitting or merging is required.

Due to the vast amount of research in image segmentation a comprehensive overview is beyond the scope of this article. We briefly review some well-known segmentation methods. Caselles et al. [15] introduced a geodesic active contour model by minimizing the related energy

$$
E_{g}[c]=\alpha \int_{0}^{1}\left\|c_{z}(z)\right\|^{2} \mathrm{~d} z+\int_{0}^{1} g\left(\left\|\nabla u_{0}^{\sigma}(c(z))\right\|\right)^{2} \mathrm{~d} z
$$

in which $u_{0}^{\sigma}$ denotes a regularized version of $u_{0}$ and $\sigma>0$ plays the role of a scale parameter. It turns out that the minimization of this energy is equivalent to finding geodesics with respect to a space-dependent isotropic metric $g$, by minimization of the energy

$$
\tilde{E}_{g}[c]=2 \alpha^{-2} \int_{0}^{1} g\left(\left\|\nabla u_{0}^{\sigma}(c(z))\right\|\right)^{2}\left\|c_{z}(z)\right\| \mathrm{d} z=\int_{\Gamma_{c}} g \mathrm{~d} A
$$

In contrast to (1.1), this functional is completely intrinsic, i. e., the energy does not depend on the specific parametrization of the contour but only on the geometry of the curve itself. The notion of equivalence of the minimization of $(1.2)$ and $(1.3)$ has been made precise by Aubert and Blanc-Féraud [2], who derived that under some mild conditions on $g$, for all piecewise $C^{1}$ Jordan-curves, there exists a neighborhood in which the steepest descent direction of $(1.2)$ also decreases $(1.3)$ and vice versa. Ring and Hintermüller introduced a Newton-type optimization technique of this problem in [33]. An extension of the geodesic contour model, that aligns the contour with the morphology of the input image in the sense of the Hildreth-Marr edge detector was introduced by Kimmel and Bruckstein [39. The energy (1.3) allows to formulate the segmentation problem by modeling the metric factor $g$. Despite its conceptual attractiveness, this model does not directly address preservation of features, since $g$ does only depend on extrinsic factors and not the local geometry. 
A technique due to Mumford \& Shah [43] involves minimization of the energy

$$
E_{M S}[u, \Gamma]:=\int_{\Omega}\left(u-u_{0}\right)^{2} \mathrm{dx}+\beta \int_{\Omega \backslash \Gamma}\|\nabla u\|^{2} \mathrm{dx}+\alpha \mathscr{H}^{d-1}(\Gamma),
$$

where $\mathscr{H}^{d-1}$ denotes the $d$-1-dimensional Hausdorff-measure. This method combines edge-preserving image denoising with segmentation in such a way that the discontinuity set $\Gamma$ of the reconstructed function $u$ divides the image into separate homogeneous regions. Later the regularization term $\mathscr{H}^{d-1}(\Gamma)$ has been extended to also take into account the curvature (Mumford-Shah-Euler \& Mumford-Shah-Nitzberg) [44. Chan and Vese [16] have formulated a piecewise constant variant of this model, in which the discontinuity set is represented by a level set function.

All of these methods incorporate curve regularization via length measurement (possibly non-homogeneously weighted in space), hence none of them directly addresses the problem of segmenting objects with geometric features: the internal energy will always result in a smoothing of sharp angles of the boundary contour.

It would appear natural to leverage formal descriptions of anisotropies from materials science, where crystalline structures are expressed by so called Wulff-shapes [59. If a description of the local morphology, in terms of normals and anisotropy, is known at each point of the image, the minimization of an anisotropic area functional could be guided by prescribing a Wulff-shape 22. Numerical methods have already been developed for problems as anisotropic mean curvature flow [24, 5], anisotropic ROF [31] or surface diffusion [25, 11, 19]. However, since the automatic detection of Wulff-shapes is a difficult problem, we focus on automatic feature preservation that is guided by the geometry of the shape variable itself, allowing the curve to adjust itself to features without prior knowledge of the morphology.

Our approach is motivated by low-curvature image simplifiers (LCIS) originating in a paper by Tumblin \& Turk [56] and further developed by Bertozzi \& Greer, who have developed a well-posedness theory and devised a Laplacian limiting scheme (see [6] for details). The key observation is that the fourth-order PDE

$$
u_{t}+\operatorname{div}(g(\Delta u) \nabla \Delta u)=0
$$

produces solutions that dynamically smooths noise while preferring locally linear shapes, resulting in regions of constant slope separated by small regions of rapidly changing curvature. In particular they were able to prove that 1.5 has globally smooth solutions from smooth initial data in one dimension for the specific functional $g$ proposed by Perona and Malik [45], $g(s)=\left(1+\frac{s^{2}}{\eta^{2}}\right)^{-1}$, using nonlinear entropy estimates motivated by related equations from lubrication theory. Since the results were proved for a periodic geometry it is natural to consider a geometric variant of this model for a self-evolving closed curve in the plane. We note that global wellposedness depends on details of the nonlinearity in $g$ and there are some subtleties that are discussed in this paper and in [7. Furthermore, the influence of the surface metric makes it difficult to transfer the results to the geometric case. Nevertheless our point here is to use the fact that 1.5 has been shown to be a highly effective denoising model for piecewise linear signals, to motivate a geometry-based curve evolution model that is effective for segmenting objects with corners (the geometric analogue of a piecewise linear function).

The solution $u$ is smooth, hence the notion of corners is understood in an infinitesimal sense. The dynamics can be combined with a basic $L^{2}$ fidelity term, leading to 
very effective denoising of piecewise linear data. In contrast, second-order adaptive filtering techniques like Perona-Malik [45] diffusion filtering or TV-based techniques like Rudin-Osher-Fatemi (ROF) filtering [47 typically develop some staircaising in the resulting image which can be undesirable in continuous non-constant regions. We note that (1.5) is a gradient flow of a non-quadratic energy functional on $\Delta u$ and preferably smoothes in regions of low curvature, while strong kinks, that are indicated by a large Laplacian lead to a small energy contribution. In this paper we show how to adapt this dynamics to geometric objects. In the context of variational processing of surfaces, the question of which notion of curvature to penalize naturally arises. Elsey and Esedoglu recently discovered that the minimization of the $L^{1}$-norm of the Gaussian curvature leads to a geometric analog of the ROF denoising model [30. In this paper, we will focus on mean-curvature based models that arise as analogies of LCIS and as weighted Willmore flow. We develop algorithms rather than rigorous theory. It would be interesting in future work to develop analysis for these methods, along the lines of [ 6 ] for the LCIS problem.

This paper is organized as follows. In Section 2 we introduce two approaches for corner preserving regularization of contours. Aiming at regularization strategies for a wider class of problems, we will consider both an evolution type approach and a variational approach, which is based on a general functional depending on the mean curvature. We will extend the Willmore functional and its variation to a general mean-curvature-dependent integrand in order to obtain a suitable weak formulation, that can be split into two inter-dependent second-order equations. In Section 3 we will describe in detail, how the continuous equations can be discretized with a finite element scheme. In Section 4 we describe a multiscale strategy based on inverse scale-space techniques which are especially suitable for generating coarse scale representations, that contain the main geometric features and apply them to segmentation of aerial images. Finally, we will present and discuss results for surface denoising.

2. Feature preserving geometric evolution equations. Unlike in the Euclidean case, there exists no estimator for sharp corners on manifolds that is given by first-order derivatives only (with respect to its local coordinates). This is due to the fact that the first derivatives of the parametrization characterize the first fundamental form, which is an intrinsic property.

In the following we propose geometric analogies of 1.5 . We will give an overview of two different possibilities, that appear naturally in the higher-order case, namely

- an evolution equation that is motivated by the surface diffusion equation, which is obtained by a weighted $H^{-1}$ metric for the area functional, and

- a gradient flow equation of a convex energy depending on the curvature of the moving contour which resembles a generalized Willmore flow.

2.1. Basic geometric notations. Let us first describe the basic differential geometric setting. For the sake of a more compact presentation we consider smooth closed manifolds $\Gamma$ embedded in $\mathbb{R}^{d+1}, d=1,2$. Given a countable atlas $\left\{\left(x^{\alpha}, \Omega^{\alpha}\right)\right\}_{\alpha}$ with reference domains $\Omega^{\alpha} \subset \mathbb{R}^{d}$, and the corresponding coordinate map $x^{\alpha}: \Omega^{\alpha} \rightarrow \Gamma$, the vectors $\frac{\partial}{\partial \xi_{i}^{\alpha}}, i=1, \ldots, d$, span a basis of the tangent space $T_{p} \Gamma$ at the point $p \in \Gamma$.

Tangent vectors can be interpreted as linear functionals on $C^{\infty}(\Gamma)$ :

$$
\frac{\partial}{\partial \xi_{i}^{\alpha}}(x) f:=\frac{\partial f\left(x^{\alpha}\right)}{\partial \xi_{i}^{\alpha}}(\xi), \quad x=x^{\alpha}(\xi) .
$$

On the tangent bundle $T \Gamma$, the metric $g: T_{p} \Gamma \times T_{p} \Gamma \rightarrow \mathbb{R}$ for all $p \in \Gamma$ can be defined from the embedding and subsequent identification of tangent vectors with vectors in 
$\mathbb{R}^{d+1}$ as

$$
g_{i j}=g_{\Gamma}\left(\frac{\partial}{\partial \xi_{i}^{\alpha}}, \frac{\partial}{\partial \xi_{j}^{\alpha}}\right):=\frac{\partial x^{\alpha}}{\partial \xi_{i}^{\alpha}} \cdot \frac{\partial x^{\alpha}}{\partial \xi_{j}^{\alpha}} .
$$

Since this equation describes the metric on the atlas, it also defines the entire metric $g$. The components of the inverse $g_{\Gamma}^{-1}$ are as usual denoted by $\left(g_{\Gamma}^{i j}\right)_{i j}$.

Due to countability of the atlas, the existence of a partition of unity, allows to define the integration of a function $f$ on $\Gamma$ by aggregation. Here, the volume element $\mathrm{d} A$ is given by $\sqrt{\operatorname{det} g_{\Gamma}} \mathrm{d} \xi$. This leads to a straightforward definition of the scalarproducts on $C^{0}(\Gamma)$ and $C^{0}(T \Gamma)$ :

$$
(f, g)_{\Gamma}:=\int_{\Gamma} f g \mathrm{~d} A \quad \text { and } \quad(v, w)_{T \Gamma}:=\int_{\Gamma} g_{\Gamma}(v, w) \mathrm{d} A
$$

The total differential of a function $f \in C^{1}(\Gamma)$ is a linear functional $\mathrm{d} f$, i. e.,

$$
\left\langle\frac{\partial}{\partial \xi_{i}}, d f\right\rangle:=\frac{\partial}{\partial \xi_{i}}(\xi)(f):=\frac{\partial f \circ x}{\partial \xi_{i}}(\xi), \quad x=x^{\alpha}(\xi) .
$$

The gradient $\nabla_{\Gamma} f$ is the representation of $d f$ in the metric $g$, implicitly given by

$$
g_{\Gamma}\left(\nabla_{\Gamma} f, \frac{\partial}{\partial \xi_{i}}\right)=\left\langle\frac{\partial}{\partial \xi_{i}}, d f\right\rangle \quad i=1, \ldots, d
$$

For a vector field $v$ we define the divergence $\operatorname{div}_{\Gamma} v$ as the dual operator of the gradient with respect to $g$ :

$$
\int_{\Gamma} \operatorname{div}_{\Gamma} v \varphi \mathrm{d} A:=-\int_{\Gamma} g_{\Gamma}\left(v, \nabla_{\Gamma} \varphi\right) \mathrm{d} A \quad \forall \varphi \in C^{\infty}(\Gamma) .
$$

Furthermore, the Laplace-Beltrami operator is given by $\Delta_{\Gamma}:=\operatorname{div}_{\Gamma} \nabla_{\Gamma}$. The scalar mean curvature is denoted by $h:=\operatorname{tr}(S)$.

2.2. Weighted surface diffusion. We obtain a straightforward geometric variant of 1.5 by replacing the differential operators by their corresponding intrinsically geometric counterparts and by choosing the coordinates $x$ as the free variable. The active contour $\Gamma$ is always understood as a $d$-dimensional compact and oriented manifold, which is immersed by the coordinate mapping $x: \Gamma \rightarrow \mathbb{R}^{d+1}$. We arrive at the evolution equation

$$
\begin{aligned}
x_{t}+\operatorname{div}_{\Gamma}\left(g(h) \nabla_{\Gamma} \Delta_{\Gamma} x\right) & =0, \\
x(0, \cdot) & =x_{0},
\end{aligned}
$$

which is very similar to the equation which describes the evolution of surfaces under surface diffusion where the Perona-Malik weighting function $g(s)=\left(1+\frac{s^{2}}{\eta^{2}}\right)^{-1}$ is used as a mobility, that depends on the scalar mean curvature $h$ :

$$
\begin{aligned}
x_{t}+\operatorname{div}_{\Gamma}\left(g(h) \nabla_{\Gamma} h\right) n & =0, \\
x(0, \cdot) & =x_{0} .
\end{aligned}
$$

Here, $n$ denotes the outer normal of $\Gamma$. Note, that we use $g_{\Gamma}$ for the metric and $g$ for the nonlinearity throughout this paper. 

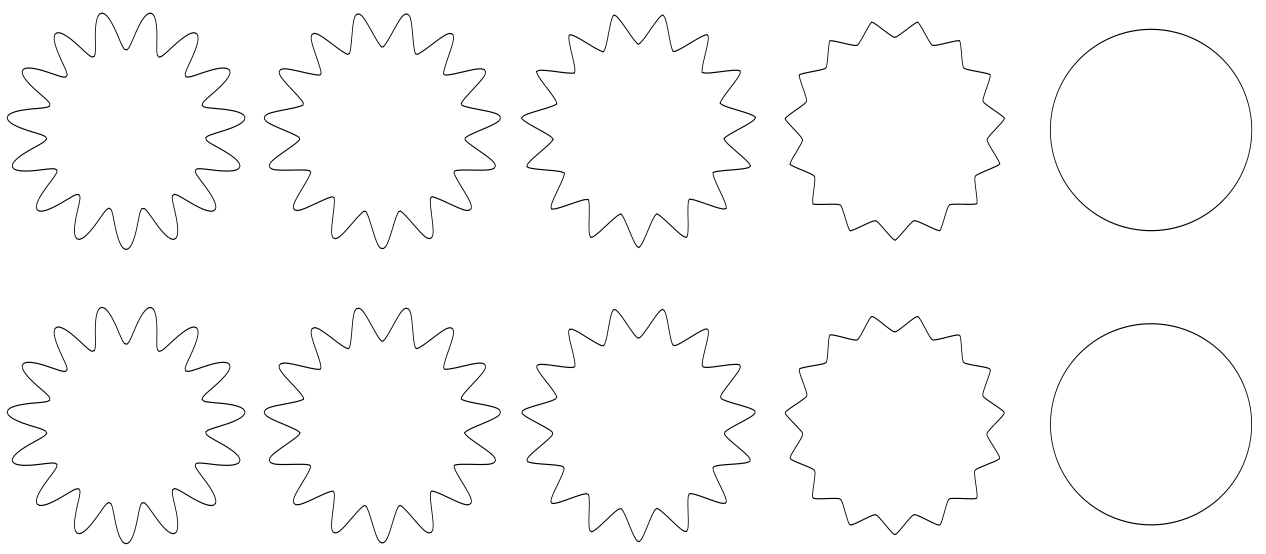

FIG. 2.1. Evolution of a simply initial geometry without external forces. Top row: weighted surface diffusion at times 0, 2.1079e-05, 4.30717e-05, 8.72062e-05 and 0.000174442 solving equation 2.8a. Bottom row: simplified version of surface diffusion 2.7a at times 0, 1.81354e-05, 3.63744e05, 7.25345e-05 and 0.000138453. The curve evolution is almost identical. The initial shape is parametrized by $x:[0,2 \pi] \rightarrow \mathbb{R}^{2}, t \mapsto r(t)(\cos (t), \sin (t))^{T}, r(t)=\frac{1}{2}+\frac{1}{10} \sin (15 t)$.

Like regular surface diffusion this weighted variant 2.8 preserves volume and decreases area, which is a desirable combination of properties for geometric regularization: since the volume is preserved, the area decrease is not achieved by uniform shrinkage, but by suppressing local oscillatory components in the curve. This is in contrast with length penality functionals that are commonly combined with an external force term as described in the introduction. The competetion of the regularization term and the driving forces would introduce a bias even for a simple shape as a circle. Due to the divergence structure, the derivation is the same as in the unweighted case ( $v$ corresponds to the velocity field):

\section{Volume preservation}

$$
\frac{d}{d t}|\Omega(t)|=\int_{\Gamma(t)} v \mathrm{~d} A=-\int_{\Gamma(t)} \operatorname{div}_{\Gamma}\left(g(h) \nabla_{\Gamma} h\right) \mathrm{d} A=\int_{\Gamma(t)} g(h) g_{\Gamma}\left(\nabla_{\Gamma} h, \nabla_{\Gamma} 1\right) \mathrm{d} A=0 .
$$

Area decrease (energy dissipation):

$$
\begin{array}{rl}
\frac{d}{d t}|\Gamma(t)|=-\int_{\Gamma(t)} v & \mathrm{~d} A \\
& =\int_{\Gamma(t)} \operatorname{div}_{\Gamma}\left(g(h) \nabla_{\Gamma} h\right) h \mathrm{~d} A=-\int_{\Gamma(t)} g(h) g_{\Gamma}\left(\nabla_{\Gamma} h, \nabla_{\Gamma} h\right) \mathrm{d} A \leq 0 .
\end{array}
$$

Unfortunately, the preservation of volume is not guaranteed for the evolution under (2.7). However, in our experiments 2.7) and 2.8 behave very similarly as indicated in Fig. 2.1] the top row of Figure 2.1 shows the behavior of weighted surface diffusion (2.8), whereas the bottom row shows the evolution under equation 2.7). The curve develops corners early by accentuating the high-curvature areas, still retains infinitesimal regularity and eventually converges to a circle. Note that the circle is stationary for both equations, whereas second order models usually continue 
to shrink, since the underlying functionals penalize area. This justifies to use 2.7 in the context of geometric regularization. The preservation of features plays a much more important role and is already a substantial improvement. In the computations we have used $\eta=1$ and approximated the curve by a polygon with 512 segments.

Furthermore, solving the exact surface diffusion equation numerically is a quite complex task, since additional equations have to be introduced to convert vectorial quantities to scalar quantities and vice versa. Furthermore, the system is numerically solved by a Schur-complement approach. The evaluation of the operator that has to be inverted in every time step involves an inversion of a discrete second order differntial operator, which makes the computation by using an iterative solver prohibitively expensive. In this paper, we are only interested in this equation for the sake of qualitative comparisons and refer to [4 for details on the isotropic case. We will see later (see Section 3) that equation (2.7) is much easier to implement and is less computationally expensive.

To use the evolution equation (2.7) in a snake evolution context, we consider

$$
x_{t}=-\operatorname{div}_{\Gamma}\left(g(h) \nabla_{\Gamma} \Delta_{\Gamma} x\right)+\gamma f_{\text {ext }}(x),
$$

where $f_{\text {ext }}: \Omega \rightarrow \mathbb{R}^{d}$ stands for the external driving force. As described before, the extrinsic force is responsible for moving the curve towards the object boundaries. Its modeling and computation highly depends on the type of input image and is a subject on its own. Frequently, such forces are designed to point towards image features, such as edges. Ideally the force is zero only on the boundary of the object to be segmented, which is in practice not achievable (or the segmentation problem would already be solved), so it introduces a dependency of the computed solution to the initial curve configuration. In Section 4.1 this will be described in more details.

Only normal movements have an influence on the shape of the evolved curve, but the external force field may point in any direction. Hence, tangential shifts may result in an undesirably uneven distribution of points along the discretized curve. In analogy to the variational approach, where it is sufficient to consider the normal variations of the energy, we rule out tangential shifts, by replacing the external force in 2.9 by its projection in normal direction:

$$
x_{t}=-\operatorname{div}_{\Gamma}\left(g(h) \nabla_{\Gamma} \Delta_{\Gamma} x\right)+\gamma(n \otimes n) f_{\text {ext }}(x) .
$$

Note that we have dropped the lower order regularization term, since the regularization is dominated by the higher order term.

2.3. Energies with mean curvature dependent densities. It is often not convenient to solely have a flow equation available. For instance, optimization techniques and step-size control rules usually require the evaluation of the full regularized functional. Furthermore, all three fundamental segmentation models mentioned in the introduction are variational methods. To fill this gap, we now consider the energy

$$
W[\Gamma]=\int_{\Gamma} G(h) \mathrm{d} A .
$$

For quadratic $G$ the above energy corresponds to the so-called Willmore-energy [40, 41, 42, 53, 52, 58, 28, 19, 20. Spheres are minimizing critical points of the Willmore energy with value $16 \pi \sqrt{1}$ Intuitively, $W[\Gamma]-16 \pi$ describes how much $\Gamma$ deviates from a sphere by measuring the amount of bending.

\footnotetext{
${ }^{1}$ Note that $h$ denotes the sum of principal curvatures, not the average.
} 

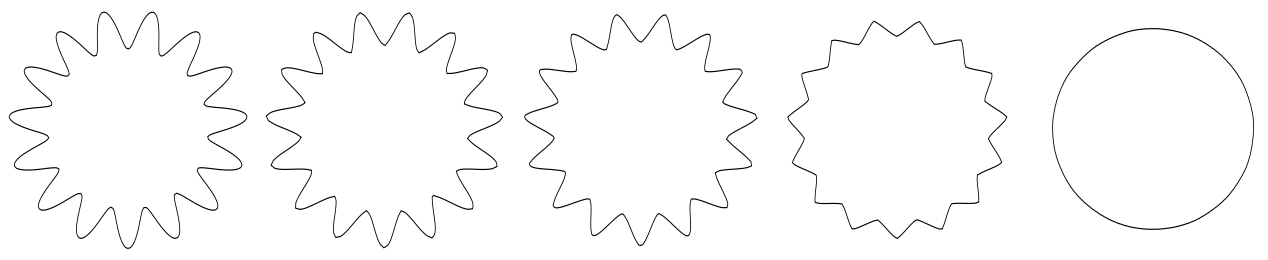

FIG. 2.2. Evolution of a simply initial geometry without external forces under gradient flow of the weighted Willmore functional at times 0, 0.6.37621e-06, 1.28998e-05, 2.58389e-05 and 5.16116e05.

The energy is entirely intrinsic, since integration takes place over $\Gamma$ and the integrand only depends on an intrinsic geometric quantity. We will see later (Sec. 2.4.2), that for closed $\Gamma$, the variation of $W$ is given by

$$
\left\langle W^{\prime}[x], \vartheta\right\rangle=\int_{\Gamma}\left(-\operatorname{div}_{\Gamma}\left(G^{\prime \prime}(h) \nabla_{\Gamma} h\right)-G^{\prime}(h)|S|^{2}+G(h) h\right) \varphi \mathrm{d} A
$$

for variation vector fields $\vartheta$ with scalar normal part $\varphi$, where $S$ denotes the Weingarten map of $\Gamma$. Hence, the corresponding evolution by gradient descent is described by the equation

$$
\partial_{t} x(t)=\left(\operatorname{div}_{\Gamma}\left(G^{\prime \prime}(h) \nabla_{\Gamma} h\right)+G^{\prime}(h)|S|^{2}-G(h) h\right) n .
$$

To obtain a sensitivity with respect to high curvatures we choose

$$
G(s):=\eta s \arctan \left(\frac{s}{\eta}\right)-\frac{1}{2} \eta^{2} \log \left(1+\frac{s^{2}}{\eta^{2}}\right) .
$$

Note that $G^{\prime \prime}(s)=\left(1+\frac{s^{2}}{\eta^{2}}\right)^{-1}=g(s)$ and that the highest-order term is the same as in the weighted surface diffusion equation (2.8a). However, due to further contributions in non-divergence form, we can no longer expect volume preservation. In the vicinity of zero, $G$ is close to quadratic and has a regularizing effect similar to Willmore flow. For $s \rightarrow \infty, G$ becomes almost linear, which leads to the preservation of strong features, since varying the argument affects the energy only marginally. This is analogous to smooth approximations of the TV functional, in the form of a Huber functional of $\|\nabla u\|$ used to obtain piecewise constant approximations [7]. Figure 2.2 shows that the qualitative properties of the evolution is very similar to 2.8a).

A simple, but useful advantage of the weighted Willmore approach, is that it can easily be incorporated into a geometric shape minimization approach. Using the above energy, one could for instance include a higher-order regularization term into the Mumford-Shah-functional, which would allow to represent discontinuity sets with sharp corners by choosing $\beta$ small:

$$
\hat{E}_{\mathrm{MS}}[u, \Gamma]:=\int_{\Omega}\left(u-u_{0}\right)^{2} \mathrm{dx}+\alpha \int_{\Omega \backslash \Gamma}\|\nabla u\|^{2} \mathrm{dx}+\beta \mathscr{H}^{d-1}(\Gamma)+\frac{1}{\gamma} \int_{\Gamma} G(h) \mathrm{d} A .
$$

As described in Section 4.1 we will use the following piecewise constant version of this model for some of our numerical experiments:

$$
E_{\mathrm{MS}}^{\mathrm{pw}}\left[c_{0}, c_{1}, \Gamma\right]:=\gamma \sum_{i=1,2} \int_{\Omega_{i}}\left(c_{i}-u_{0}\right)^{2} \mathrm{dx}+\int_{\Gamma} G(h) \mathrm{d} A,
$$


where $\bar{\Omega}=\bar{\Omega}_{1} \cup \bar{\Omega}_{2}, \Omega_{1} \cap \Omega_{2}=\emptyset$ and $\bar{\Omega}_{1} \cap \bar{\Omega}_{2}=\Gamma$.

In general, we observed in our experiments, that the denoising properties of weighted surface diffusion, the simplified variant (2.7a) and gradient flow of the weighted Willmore functional are very similar. Local oscillatory components are very quickly smoothed out, whereas the global shape is preserved for a longer time.

2.4. Variation of the weighted Willmore functional. In this section we will compute the variation of the functional $W$ and formulate a weak formulation that is suitable for a spatial finite element discretization.

2.4.1. Differential geometric tools. We will need the following lemmas. The proofs can be found in [26, which primarily addresses the anisotropic case.

Lemma 2.1. Let $x_{\epsilon}=x+\epsilon \vartheta+O\left(\epsilon^{2}\right)$ be a variation of $x \in \Gamma$ in direction of the variation vector field $\vartheta=\phi n+D x(v)$ and $h_{\epsilon}$ the mean curvature of the perturbed surface $x_{\epsilon}$. Then

$$
\left.\partial_{\epsilon} h_{\epsilon}\right|_{\epsilon}=-\Delta_{\Gamma} \varphi-|S|^{2} \varphi+g_{\Gamma}\left(\operatorname{grad}_{\Gamma} h, v\right) .
$$

Lemma 2.2 (Derivation of the area-element). Let $x_{\epsilon}=x+\epsilon \vartheta+O\left(\epsilon^{2}\right)$ be $a$ variation of $x \in \Gamma$ in direction of the variation vector field $\vartheta=\phi n+D x(v)$. Let $g_{\Gamma_{\epsilon}}$ be the fundamental form of the perturbed surface. Then the derivation of the area element is locally given by

$$
\partial_{\epsilon} \sqrt{\operatorname{det} g_{\Gamma_{\epsilon}}} d \xi=\operatorname{div}_{\Gamma} \vartheta \sqrt{\operatorname{det} g_{\Gamma}} d \xi
$$

Lemma 2.3 (Tangential and normal components of $\Delta_{\Gamma} \vartheta$ ). Let $\vartheta=\varphi n+D x(v)$ be a perturbation vectorfield on $\Gamma$, then the following identity holds:

$$
\Delta_{\Gamma} \vartheta=\underbrace{\left(\Delta_{\Gamma} \varphi-\varphi|S|^{2}\right) n}_{\in D x(T \Gamma)^{\perp}}+\underbrace{2 D n\left(\nabla_{\Gamma} \varphi\right)+\varphi D x\left(\nabla_{\Gamma} h\right)}_{\in D x(T \Gamma)}+\Delta_{\Gamma} D x(v),
$$

where the normal component of the last term is given by

$$
\left\langle\Delta_{\Gamma} D x(v), n\right\rangle n=-\left(g_{\Gamma}\left(v, \nabla_{\Gamma} h\right)+2 \operatorname{tr}(S \nabla \cdot v)\right) n .
$$

Here, $\nabla . v \in \operatorname{End}\left(T_{p} \Gamma\right)$ denotes the Riemannian connection. For further details we refer to [27]). The short notation $\vartheta=\varphi n+D x(v)$ stands for the decomposition of $\vartheta$ into the scalar normal factor $\varphi$ and the tangential component $D x(v)$.

2.4.2. First variation. From now on we will consider an immersion $x: \Gamma \rightarrow \mathbb{R}^{d}$ and formulate the energy $W$ in terms of $x$ instead of $\Gamma$. Let

$$
W[x]=\int_{\Gamma} G(h) \mathrm{d} A .
$$

Recall that we assume that $\Gamma$ is a closed manifold, in order to avoid several boundary integrals. Let us now derive the first variation of $W$ at $x$ in a perturbation vector 
field $\vartheta$ :

$$
\begin{aligned}
\left\langle W^{\prime}[x], \vartheta\right\rangle= & \left.\frac{d}{d \epsilon} W\left(x_{\epsilon}\right)\right|_{\epsilon=0} \\
\stackrel{2.18}{-} & \int_{\Gamma} G^{\prime}(h) \partial_{\epsilon} h_{\epsilon} \mathrm{d} A+\int_{\Gamma} G(h) \operatorname{div}_{\Gamma} \vartheta \mathrm{d} A \\
= & -\int_{\Gamma} G^{\prime}(h)\left(\Delta_{\Gamma} \varphi+|S|^{2} \varphi-g_{\Gamma}\left(\operatorname{grad}_{\Gamma} h, v\right)\right) \mathrm{d} A \\
& +\int_{\Gamma} G(h) \operatorname{div}_{\Gamma} \vartheta \mathrm{d} A \\
\frac{2.22}{-} & -\int_{\Gamma} G^{\prime}(h)\left(\Delta_{\Gamma} \varphi+|S|^{2} \varphi-g_{\Gamma}\left(\operatorname{grad}_{\Gamma} h, v\right)\right) \mathrm{d} A \\
& +\int_{\Gamma} \operatorname{div}_{\Gamma}(G(h) v)-G^{\prime}(h) g_{\Gamma}\left(\operatorname{grad}_{\Gamma} h, v\right)+G(h) \underbrace{\operatorname{div}(\varphi n)}_{\varphi h} \mathrm{~d} A \\
= & \int_{\Gamma}\left(-G^{\prime}(h)\left(\Delta_{\Gamma} \varphi+|S|^{2} \varphi\right)+\operatorname{div}_{\Gamma}(G(h) v)+\varphi G(h) h\right) \mathrm{d} A,
\end{aligned}
$$

where we have used the relation

$$
\begin{aligned}
\operatorname{div}_{\Gamma}(G(h) v) & =G(h) \operatorname{div}_{\Gamma} v+g_{\Gamma}\left(\operatorname{grad}_{\Gamma} G(h), v\right) \\
& =G(h) \operatorname{div}_{\Gamma} v+G^{\prime}(h) g_{\Gamma}\left(\operatorname{grad}_{\Gamma} h, v\right) .
\end{aligned}
$$

The first term becomes

$$
\begin{aligned}
-\int_{\Gamma} G^{\prime}(h) \Delta_{\Gamma} \varphi \mathrm{d} A & =-\int_{\Gamma} G^{\prime}(h) \operatorname{div}_{\Gamma}\left(\nabla_{\Gamma} \varphi\right) \mathrm{d} A \\
& =\int_{\Gamma} g_{\Gamma}\left(\nabla_{\Gamma} G^{\prime}(h), \nabla_{\Gamma} \varphi\right) \mathrm{d} A \\
& =-\int_{\Gamma} \operatorname{div}_{\Gamma}\left(\nabla G^{\prime}(h)\right) \varphi \mathrm{d} A \\
& =-\int_{\Gamma} \operatorname{div}_{\Gamma}\left(G^{\prime \prime}(h) \nabla_{\Gamma} h\right) \varphi \mathrm{d} A,
\end{aligned}
$$

and we obtain

$$
\left\langle W[x]^{\prime}, \vartheta\right\rangle=\int_{\Gamma}\left(-\operatorname{div}_{\Gamma}\left(G^{\prime \prime}(h) \nabla_{\Gamma} h\right)-G^{\prime}(h)|S|^{2}+G(h) h\right) \varphi \mathrm{d} A .
$$

LEMma 2.4 (First Variation, preliminary weak form). Let $x_{\epsilon}=x+\epsilon \vartheta+O\left(\epsilon^{2}\right)$ be a variation of $x \in \Gamma$ in direction of the variation vector field $\vartheta=\varphi n+D x(v)$. Then the first variation of the weighted Willmore functional can be written as

$$
\begin{aligned}
\left\langle W_{g}^{\prime}[x], \vartheta\right\rangle=\int_{\Gamma}\left\langle-G^{\prime}(h) n, \Delta_{\Gamma} \vartheta\right\rangle d A-2 & \int_{\Gamma} G^{\prime}(h) D x\left(\nabla_{\Gamma} n\right): D x\left(\nabla_{\Gamma} \vartheta\right) d A \\
& +\int_{\Gamma} G(h) D x\left(\nabla_{\Gamma} x\right): D x\left(\nabla_{\Gamma} \vartheta\right) d A
\end{aligned}
$$


Proof.

$$
\begin{aligned}
\left\langle W_{g}^{\prime}[x], \vartheta\right\rangle & =\left.\int_{\Gamma} G^{\prime}(h) \partial_{\epsilon} h_{\epsilon}\right|_{\epsilon=0} \mathrm{~d} A+\int_{\Gamma} G(h) \operatorname{div}_{\Gamma} \vartheta \mathrm{d} A \\
& =-\int_{\Gamma} G^{\prime}(h)\left(\Delta_{\Gamma} \varphi+|S|^{2} \varphi-g_{\Gamma}\left(\nabla_{\Gamma} h, v\right)\right) \mathrm{d} A \\
& +\int_{\Gamma} G(h) D x\left(\nabla_{\Gamma} x\right): D x\left(\nabla_{\Gamma} \vartheta\right) \mathrm{d} A .
\end{aligned}
$$

We know from the proof of Thm. 71 in [26] using $\gamma(n)=\|n\|$ and hence $a_{\gamma}=\mathbb{1}$ : $T \Gamma \rightarrow T \Gamma$ that

$$
D x\left(\nabla_{\Gamma} n\right): D x\left(\nabla_{\Gamma} \vartheta\right)=\varphi|S|^{2}+\operatorname{tr}(S \nabla . v) .
$$

Using this and Lemma 2.3 we obtain:

$$
\begin{aligned}
\left\langle-G^{\prime}(h) n, \Delta_{\Gamma} \vartheta+2 n D x\left(\nabla_{\Gamma} n\right): D x\left(\nabla_{\Gamma} \vartheta\right)\right\rangle= & -G^{\prime}(h)\left(\Delta_{\Gamma} \varphi-\varphi|S|^{2}\right) \\
& +G^{\prime}(h) g_{\Gamma}\left(v, \nabla_{\Gamma} h\right) \\
& +2 G^{\prime}(h) \operatorname{tr}(S \nabla \cdot v) \\
& -2 G^{\prime}(h)|S|^{2} \\
& -2 G^{\prime}(h) \operatorname{tr}(S \nabla \cdot v) \\
= & -G^{\prime}(h)\left(\Delta_{\Gamma} \varphi+\varphi|S|^{2}\right) \\
& +G^{\prime}(h) g_{\Gamma}\left(v, \nabla_{\Gamma} h\right)
\end{aligned}
$$

which is the desired result.

THEOREM 2.5 (First Variation, weak form). Let $x_{\epsilon}=x+\epsilon \vartheta+O\left(\epsilon^{2}\right)$ be a variation of $x \in \Gamma$ in direction of the variation vector field $\vartheta=\varphi n+D x(v)$. Then using the variable substitution $w=-G^{\prime}(h) n$ the first variation of the weighted Willmore functional can be written as

$$
\begin{aligned}
\left\langle W_{g}^{\prime}[x], \vartheta\right\rangle=\int_{\Gamma} D x\left(\nabla_{\Gamma} w\right): D x\left(\nabla_{\Gamma} \vartheta\right) d A & \\
& -2 \int_{\Gamma}\left\langle n_{l} D x\left(\nabla_{\Gamma} w_{l}\right), n_{i} D x\left(\nabla_{\Gamma} \vartheta_{i}\right)\right\rangle d A \\
& \quad+\int_{\Gamma} G(h) D x\left(\nabla_{\Gamma} x\right): D x\left(\nabla_{\Gamma} \vartheta\right) d A .
\end{aligned}
$$

Proof. We further analyze the following terms from Lemma 2.4

$$
\int_{\Gamma}\left\langle G^{\prime}(h) n, \Delta_{\Gamma} \vartheta\right\rangle \mathrm{d} A+\int_{\Gamma}\left\langle G^{\prime}(h) n, 2 n D x\left(\nabla_{\Gamma} n\right): D x\left(\nabla_{\Gamma} \vartheta\right)\right\rangle \mathrm{d} A=:(I)+(I I) .
$$

By integrating by parts, we obtain

$$
\begin{aligned}
(I) & =\int_{\Gamma}\left\langle G^{\prime}(h) n, \Delta_{\Gamma} \vartheta\right\rangle \mathrm{d} A \\
& =-\int_{\Gamma} g_{\Gamma}\left(\nabla_{\Gamma}\left(G^{\prime}(h) n_{k}\right), \nabla_{\Gamma} \vartheta_{k}\right) .
\end{aligned}
$$


The second term can be transformed the following way

$$
\begin{aligned}
(I I)= & 2 \int_{\Gamma} G^{\prime}(h) D x\left(\nabla_{\Gamma} n\right): D x\left(\nabla_{\Gamma} \vartheta\right) \mathrm{d} A \\
= & 2 \int_{\Gamma} g_{\Gamma}\left(\left\langle n, G^{\prime}(h) n\right\rangle \nabla_{\Gamma} n, \nabla_{\Gamma} \vartheta\right) \mathrm{d} A \\
= & 2 \int_{\Gamma} g_{\Gamma}\left(\nabla_{\Gamma}\left(G^{\prime}(h) n_{i}\right), \nabla_{\Gamma} \vartheta_{i}\right) \mathrm{d} A \\
& -2 \int_{\Gamma} g_{\Gamma}\left(n_{l}\left(\nabla_{\Gamma} G^{\prime}(h) n_{l}\right) n_{i}, \nabla_{\Gamma} \vartheta_{i}\right) \mathrm{d} A \\
& -2 \int_{\Gamma} \underbrace{\left.g_{\Gamma}\left(\left(\nabla_{\Gamma} n_{l}\right) G^{\prime}(h) n_{l} n_{k}, \nabla_{\Gamma} \vartheta_{k}\right)\right)}_{=0} \mathrm{~d} A
\end{aligned}
$$

Remark Observe that the combination of the terms leads to a change in sign of the term

$$
\int_{\Gamma} g_{\Gamma}\left(\nabla_{\Gamma}\left(G^{\prime}(h) n_{k}\right), \nabla_{\Gamma} \vartheta_{k}\right) \mathrm{d} A
$$

The forward diffusion of the highest order operator is hence "hidden" in the term involving the normal projection.

3. Numerical approximation. In this section, we will describe the numerical schemes that we have used for the discretization of the previously introduced geometric evolution equations. Both variants directly lead to weak formulations that allow the discretization by a finite element method.

3.1. Discretization in space. We consider a finite element discretization with a Lagrange basis of piecewise affine elements on the discrete interface $\Gamma_{h}$ and define the following general forms of mass and stiffness matrices:

$$
\begin{aligned}
\mathbf{M}_{h}[\omega] & :=\left(\int_{\Gamma_{h}} \omega I_{h}\left(\Phi_{i} \Phi_{j}\right) \mathrm{d} A\right)_{1 \leq i \leq n, 1 \leq j \leq n} \\
\mathbf{L}[\omega] & :=\left(\int_{\Gamma_{h}} \omega \nabla_{\Gamma} \Phi_{i} \cdot \nabla_{\Gamma} \Phi_{j} \mathrm{~d} A\right)_{1 \leq i \leq n, 1 \leq j \leq n} \\
\mathbf{L}[A] & :=\left(\mathbf{L}\left[A_{i j}\right]\right)_{1 \leq i \leq d, 1 \leq j \leq d}
\end{aligned}
$$

Here, $I_{h}: C^{0}\left(\Gamma_{h}\right) \rightarrow \mathcal{V}^{h}$ stands for the nodal interpolation operator, which implies that the so called lumped mass matrix $\mathbf{M}_{h}$ is diagonal and can easily be inverted 55. In order to calculate the elements of the stiffness matrices, we consider for each triangle $T$ a reference triangle $\hat{T} \in \mathbb{R}^{d}$. For $d=2$, we choose $\xi^{0}=(0,0), \xi^{1}=(1,0)$ and $\xi^{2}=(0,1)$. The local chart $X$ is then given by a simple affine map from $\hat{T}$ onto $T$, which maps the nodes $\xi^{i}$ onto the corresponding nodes $P^{i} \in T$, and hence the local first fundamental form is given by

$$
g_{i j}=\frac{\partial X}{\partial \xi_{i}} \cdot \frac{\partial X}{\partial \xi_{j}}, \quad \frac{X}{\partial \xi_{i}}=P^{i}-P^{0}
$$

From the definition of the gradient 2.5, we deduce the local representation

$$
\nabla_{\Gamma_{h}} \Phi^{l}=\sum_{i, j} g^{i j} \frac{\partial \Phi^{l}}{\partial \xi_{j}}\left(P^{i}-P^{0}\right), \quad\left(\begin{array}{c}
\frac{\partial \Phi^{l}}{\partial \xi_{1}} \\
\frac{\partial \Phi^{l}}{\partial \xi_{2}}
\end{array}\right)=\left(\begin{array}{c}
-1 \\
-1
\end{array}\right),\left(\begin{array}{l}
1 \\
0
\end{array}\right),\left(\begin{array}{l}
0 \\
1
\end{array}\right)
$$


if we consider nodal basis functions $\Phi^{l} \in \mathcal{V}^{h}$. For weights $\omega$ which are constant for each triangle, e. g., functions depending on the gradient of a function $f_{h} \in \mathcal{V}^{h}$, the entries of the stiffness matrix (3.2) are given by

$$
\mathbf{L}[\omega]_{i j}=|T| \omega_{T} \nabla_{\Gamma_{h}} \Phi^{i} \cdot \nabla_{\Gamma_{h}} \Phi^{j} .
$$

3.2. Discretization of Variant I. In the following, we will describe a simple discretization scheme for the one-dimensional case of problem (2.7). We observe, that 2.10 can be written as a coupled system of two equations:

$$
\begin{aligned}
x_{t} & =\operatorname{div}_{\Gamma}\left(g(h) \nabla_{\Gamma} y\right)+\gamma(n \otimes n) f_{\text {ext }}(x) \\
y & =-\Delta_{\Gamma} x
\end{aligned}
$$

which yields the weak formulation

$$
\begin{aligned}
\left(x_{t}, \vartheta\right)_{\Gamma(t)}+\left(g(h) \nabla_{\Gamma} y, \nabla_{\Gamma} \vartheta\right)_{T \Gamma(t)} & =\gamma\left((n \otimes n) f_{\mathrm{ext}}(x), \vartheta\right)_{\Gamma(t)} \\
(y, \psi)_{\Gamma(t)} & =\left(\nabla_{\Gamma} x, \nabla_{\Gamma} \psi\right)_{T \Gamma(t)}
\end{aligned}
$$

for all $\vartheta, \psi \in C^{\infty}(\Gamma(t))$.

For the discretization in time we consider a first-order difference quotient approximation of $x_{t}$, i. e.,

$$
x_{t} \approx \frac{x^{k+1}-x^{k}}{\tau} \quad \text { with } \quad x^{k}:=x(k \tau) .
$$

We choose a semi-implicit scheme in time and obtain

$$
\begin{aligned}
\left(x^{k+1}-x^{k}, \vartheta\right)_{\Gamma(t)}+\tau\left(g\left(h^{k}\right) \nabla_{\Gamma} y^{k+1}, \nabla_{\Gamma} \vartheta\right)_{T \Gamma(t)} & =\tau \gamma\left(\left(n^{k} \otimes n^{k}\right) f_{\mathrm{ext}}\left(x^{k}\right), \vartheta\right)_{\Gamma(t)}, \\
\left(y^{k+1}, \psi\right)_{\Gamma(t)} & =\left(\nabla_{\Gamma} x^{k+1}, \nabla_{\Gamma} \psi\right)_{T \Gamma(t)},
\end{aligned}
$$

again for all test functions. As usual we now restrict the problem to the finite dimensional space $\mathcal{V}^{h}$ and denote the discrete representations of a continuous functions, obtained for example by projection, by capital letters. The coordinate vector of $y$ is given by $\bar{Y}=\mathbf{L} \bar{X}$. Note that the mean curvature lags behind from the previous time-step. The dependence of the differential operators on the metric is also treated explicitly. In terms of matrix and vector representations, this can be written as

$$
\left(\mathbf{M}_{h}+\tau \mathbf{L}\left[g\left(H^{k}\right)\right] \mathbf{M}_{h}^{-1} \mathbf{L}\right) \bar{X}^{k+1}=\mathbf{M}_{h} \bar{X}^{k}+\tau \gamma \mathbf{M}_{h}\left[N^{k} \otimes N^{k}\right] F_{\text {ext }}^{-}\left(X^{k}\right) .
$$

For one-dimensional contours, this system can be solved directly by a combination of the Sherman-Morrison method and Thomas' algorithm for banded matrices on periodic domains. In the case of surfaces, one can choose an iterative method such as a SSOR-preconditioned conjugate gradient solver, since the matrix on the left hand side is symmetric positive definite.

3.3. Discretization of Variant II: Weighted Willmore flow. Let us now describe the discretization of the gradient flow of the weighted Willmore functional using the weak formulation of Theorem 2.5 .

Following the approach for the discretization scheme of Rusu 48 for isotropic Willmore-flow and the implementation of Diewald [26] in the anisotropic case, we treat the term which depends on the normal of the surface explicitly and the other terms at 
least semi-implicitly. We especially want to treat the highest-order term implicitly by solving a Newton iteration or semi-implicitly by solving a single Newton-step during each time-step. We will treat the coefficient $G(h)$ of the second-order term explicitly.

More precisely, we apply the following time stepping scheme:

$$
\begin{aligned}
\int_{\Gamma} \frac{x^{k+1}-x^{k}}{\tau} \mathrm{d} A= & -\int_{\Gamma} D x\left(\nabla_{\Gamma}\left(w^{k+1}\right)\right): D x\left(\nabla_{\Gamma} \vartheta\right) \mathrm{d} A \\
& +2 \int_{\Gamma}\left\langle n_{l} D x\left(\nabla_{\Gamma} w_{l}^{k}\right), n_{i} D x\left(\nabla_{\Gamma} \vartheta_{i}\right)\right\rangle \mathrm{d} A \\
& -\int_{\Gamma} G\left(h^{k}\right) D x\left(\nabla_{\Gamma} x^{k+1}\right): D x\left(\nabla_{\Gamma} \vartheta\right) \mathrm{d} A .
\end{aligned}
$$

Note that $w^{k+1}=-G\left(h^{k+1}\right) n$ depends nonlinearly on $x^{k+1}$. After a straightforward linearization we obtain thanks to sufficient smoothness of $G$ the following Newton iteration scheme for $w^{k+1}$ :

$$
w_{j+1}^{k+1}=\underbrace{-G^{\prime \prime}\left(h_{j}^{k+1}\right)\left(h_{j+1}^{k+1}-h_{j}^{k+1}\right) n}_{=: w_{j+1,1}^{k+1}} \underbrace{-G^{\prime}\left(h_{j}^{k+1}\right) n}_{=w_{j, 2}^{k+1}}
$$

for $j=0, \ldots$ and $h_{0}^{k+1}:=h^{k}$. Let us now express $w_{j+1,1}^{k+1}$ and $w_{j, 2}^{k+1}$ in a weak sense:

$$
\begin{aligned}
\int_{\Gamma} \frac{w_{j+1,1}^{k+1} \vartheta}{G^{\prime \prime}\left(h_{j}^{k+1}\right)} \mathrm{d} A & =\int_{\Gamma}-\left(h_{j+1}^{k+1}-h_{j}^{k+1}\right) n \mathrm{~d} A=\int_{\Gamma}\left(\Delta_{\Gamma} x_{j+1}^{k+1}-\Delta_{\Gamma} x_{j}^{k+1}\right) \mathrm{d} A \\
& =-\int_{\Gamma} D x\left(\nabla_{\Gamma}\left(x_{j+1}^{k+1}-x_{j}^{k+1}\right)\right): D x\left(\nabla_{\Gamma} \vartheta\right) \mathrm{d} A \quad \forall \vartheta \in H^{1}\left(\Gamma, \mathbb{R}^{d}\right) \\
\int_{\Gamma} w_{j, 2}^{k+1} \psi \mathrm{d} A & =\int_{\Gamma}-G^{\prime}\left(h_{j}^{k+1}\right) n \psi \mathrm{d} A \quad \forall \psi \in H^{1}\left(\Gamma, \mathbb{R}^{d}\right)
\end{aligned}
$$

After restricting the problem to the discrete finite element space, the two equations are given in matrix form as follows:

$$
\begin{aligned}
\bar{W}_{j+1}^{k+1} & =-\left(\mathbf{M}_{h}\left[G^{\prime \prime}\left(H_{j}^{k+1}\right)^{-1}\right]\right)^{-1} \mathbf{L} \bar{X}_{j+1}^{k+1}+\left(\mathbf{M}_{h}\left[G^{\prime \prime}\left(H_{j}^{k+1}\right)^{-1}\right]\right)^{-1} \mathbf{L} \bar{X}_{j}^{k+1}+\bar{W}_{j}^{k+1}, \\
\mathbf{M}_{h} \bar{X}_{j+1}^{k+1} & =-\tau \mathbf{L} W_{j+1}^{k+1}-\tau \mathbf{L}\left[G\left(H^{k}\right)\right] X_{j+1}^{k+1}+\mathbf{M}_{h} \bar{X}^{k}+\tau 2 \mathbf{L}\left[N^{k} \otimes N^{k}\right] W^{k}
\end{aligned}
$$

Here $N^{k}$ denotes the normal of the discrete configuration $\Gamma^{k}$. By substituting (3.9) into 3.10 and shifting all explicitly treated terms to the right hand side, we obtain the following discrete system:

$$
\begin{aligned}
& \mathbf{M}_{h} \bar{X}_{j+1}^{k+1}+\tau \mathbf{L}\left(\mathbf{M}_{h}\left[G^{\prime \prime}\left(H_{j}^{k+1}\right)^{-1}\right]\right)^{-1} \mathbf{L} \bar{X}_{j+1}^{k+1}+\tau \mathbf{L}\left[G\left(H^{k}\right)\right] \bar{X}_{j+1}^{k+1} \\
& =\mathbf{M}_{h} \bar{X}_{j}^{k+1}+\tau \mathbf{L}\left[\left(\mathbf{M}_{h}\left[G^{\prime \prime}\left(H_{j}^{k+1}\right)^{-1}\right]\right)^{-1} \mathbf{L} \bar{X}_{j}^{k+1}-\bar{W}_{j}^{k+1}\right]+\tau 2 \mathbf{L}\left[N^{k} \otimes N^{k}\right] \bar{W}^{k}
\end{aligned}
$$

4. Numerical experiments. In this section we will describe three different application scenarios, namely segmentation of aerial images and surface processing. 
4.1. Segmentation of objects with sharp corners. We have applied the proposed regularization to segmentation of Kanizsa's triangle and real-world satellite images, aiming at the segmentation of man-made objects with sharp corners, in particular on the detection of buildings. Figures 4.1 and 4.2 show the segmentation of two such objects.

In real-life applications the external forces usually give rise to a serious nonconvexity of the problem. A natural approach to overcome this problem is to consider multi scale techniques for the generation of the external forces in addition to the internal regularization. A common multi scale approach for the regularization of variational problems is a coarse-to-fine strategy, which can be understood as a choicecriterion for picking a meaningful solution of the large space of local minima. One first computes the solution to a modified problem in which the non-convexity is strongly regularized and then aims to follow the path of solution as more detail is added. Even though this technique does usually not give any guarantees on computing the global minimum, the solutions that are computed this way have a very good chance of attaining a significantly lower energy value than that of the original problem. Naturally, it is crucial to approach the unregularized problem iteratively. This avoids having to chose a fixed scale parameter for preprocessing, which would always lead to loss of fine-scale information.

4.1.1. Inverse scale space techniques. One of the most basic coarse-to-fine scale-space technique consists in applying a linear filter such as linear diffusion on the initial image and to successively refine the scale. Even though one can get rid of most of the irrelevant and undesirable background patterns, strong features marked by edges will be blurred equally. We consider inverse scale-space techniques that are motivated by the Bregman-type iterations of the ROF-regularization with a $L^{1}$ fidelity term [12. In case of the TV- $L^{1}$ denoising model, the Bregman-iteration leads to a sequence $u_{k}$ by successively computing

$$
u_{k}=\underset{u}{\arg \min }\|u\|_{B V}+\lambda\left\|v_{k-1}+f-u\right\|_{L^{1}}, \quad k \geq 0,
$$

where $f=u_{k}+v_{k}$ and $v_{0}=0$. By interpreting the change of $v$ as an approximation to the time derivative $v_{t}$, Burger et al. [12] obtain a relaxed continuous inverse scale space formulation, which for the TV- $L^{1}$ denoising model, leads to the following evolution of the coupled system

$$
u_{t}=\operatorname{div}\left(\frac{\nabla u}{|\nabla u|}\right)+\lambda(\operatorname{sign}(f-u)+v), \quad v_{t}=\alpha \operatorname{sign}(f-u) .
$$

This flow yields a natural inverse scale, that starts at a very coarse scale - typically the mean of the initial image - and eventually converges back to the original image. However, already on coarse scales, the edges are remarkably well preserved and are thus a very suitable choice for the input of the segmentation process. We have chosen $\lambda=10^{4}$ and $\alpha=10^{3}$ for our experiments.

4.1.2. Ambrosio-Tortorelli approximation of the edge-map. The generation of a suitable external source term is a topic unto itself. We will rely on a multiscale approach for the generation of the edge map as well, since the regularization of the external force leads to a rounding effect on the corners of the objects. On the other hand, the energy landscape on the coarse initial scale has a heavily reduced amount of local minima. 
We considered the phase-field approximation of the Mumford-Shah functional of Ambrosio \& Tortorelli [1] for the computation of an edge map $w_{\epsilon}: \Omega \rightarrow[0,1]$, which is close to zero on edge sets and one elsewhere. More precisely, we follow the finite element discretization approach of Bourdin \& Chambolle [9] to minimize the energy

$$
\begin{aligned}
E_{\mathrm{A} T}[u, w]=\frac{\lambda_{\mathrm{AT}}}{2} \int_{\Omega}\left(u-u_{\mathrm{ISS}}(t)\right)^{2} \mathrm{dx} & +\frac{1}{2} \int_{\Omega}\left(w^{2}+k_{\epsilon}\right)\|\nabla u\|^{2} \mathrm{dx} \\
& +\frac{\nu_{\mathrm{AT}}}{2} \int_{\Omega}\left(\epsilon\|\nabla w\|^{2}+\frac{(1-w)^{2}}{4 \epsilon}\right) \mathrm{dx},
\end{aligned}
$$

where the weight $\lambda_{\mathrm{AT}}>0$ controls the fidelity of $u$ to $u_{\mathrm{ISS}}$, the solution of $(4.2)$. The parameter $\nu_{\mathrm{AT}}$ controls the phase-field approximation of the length term $\mathscr{H}^{d-1}(\Gamma)$, while $\epsilon$ controls the width of the profile of the phase field function $w$. We have set $\lambda_{\mathrm{AT}}=10^{4}$ and $\nu_{\mathrm{AT}}=2$. This allows us to pursue the minimization using coarse to fine edge indicators by initially choosing a large value for epsilon and reducing it until it is in the order of the grid size. The small and positive parameter $k_{\epsilon}$ ensures strong ellipticity of the coupled system. The negative gradient of the phase field function can then be used as the external driving force for the snake evolution equation.

4.1.3. Iterative procedure for controlling sensitivity to high curvature. In the early stage of the segmentation, the edge preserving property of the segmentation process is not yet important. It could even be misleading, since the contour should ideally first capture only the approximate shape of the segment. Using a very low sensitivity with respect to the curvature in the beginning and a higher sensitivity during the final stage has in our experiments shown to lead to better results in the overall segmentation process.

In the iterative segmentation procedure we aim to choose an appropriate coupling for the curvature sensitivity, the scale of the input image and the phase-field parameter $\epsilon$. The iteration is summarized in Algorithm 1

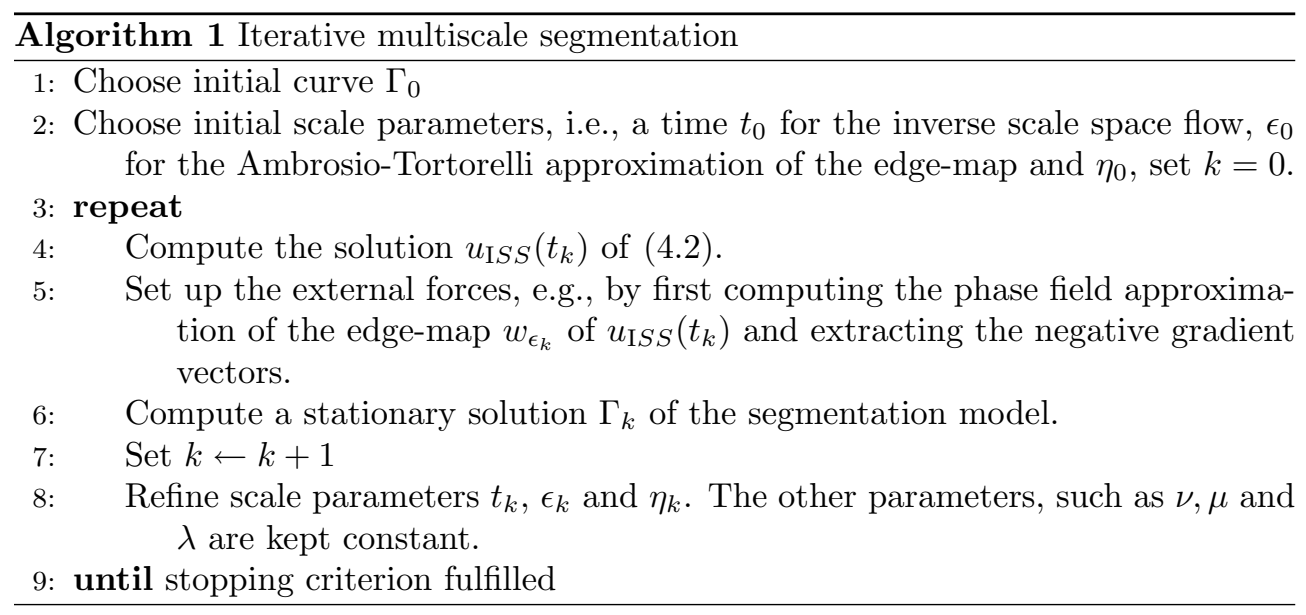

The external force computation in line 5 is only an example of possible approaches. This step opens a wide range of modeling possibilities, including interactive tools to influence the flow in complicated problem scenarios, where an automatic segmentation may fail.

Figure 4.1 shows an example on which we perform the scale space procedure. Figure 4.1(a) shows, the original image, 4.1(b) the TV- $L^{1}$ filtered image, 4.1(c) the 


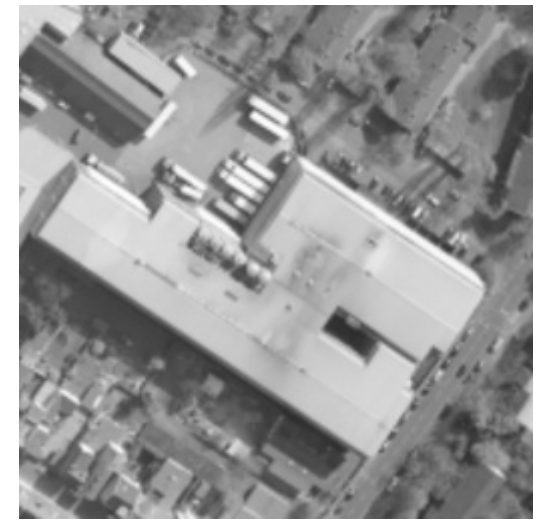

(a) original image

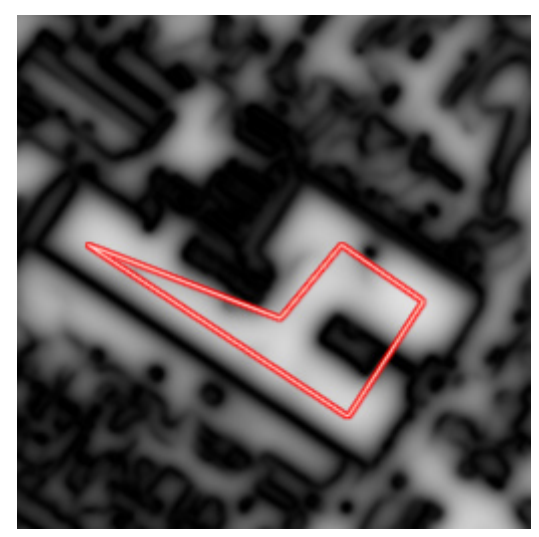

(c) initial edge map, $\epsilon=0.1$

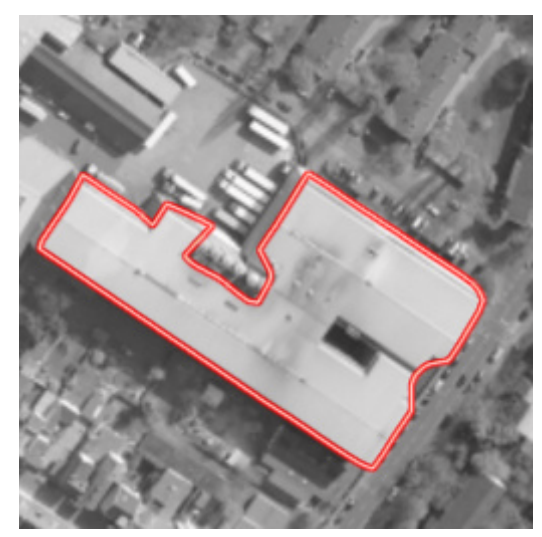

(e) final result, $\epsilon=0.002, \eta=2$

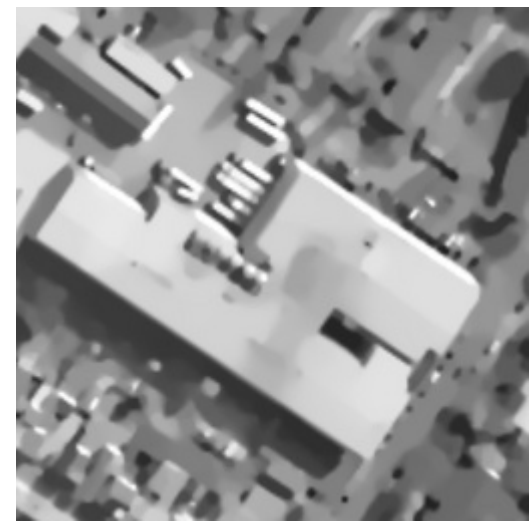

(b) applying TV- $L^{1}$ ISS filtering

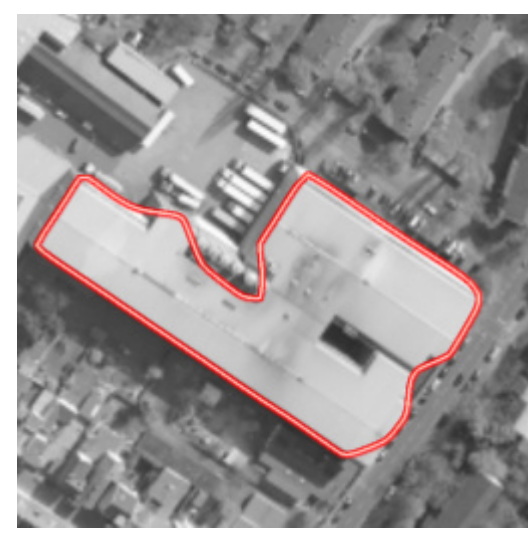

(d) intermediate stage of evolution

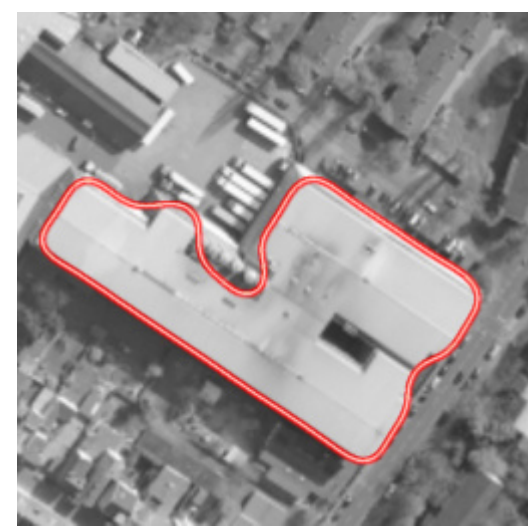

(f) result without curvature weight

FIG. 4.1. Segmentation of a satellite image of a depot building using equation 2.10

initial edge-map, followed by different stages of the evolution with (shown in Figs. 4.1(d) and 4.1(e) and without curvature dependent weight (shown in Fig. 4.1(f)]. Let us describe the whole process in more detail. As already mentioned above, the 


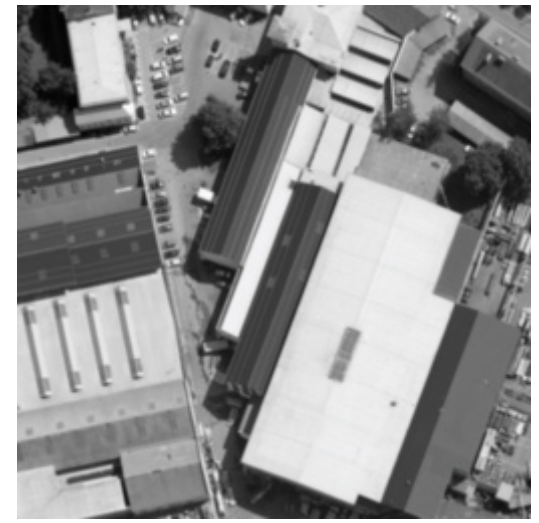

(a) original image

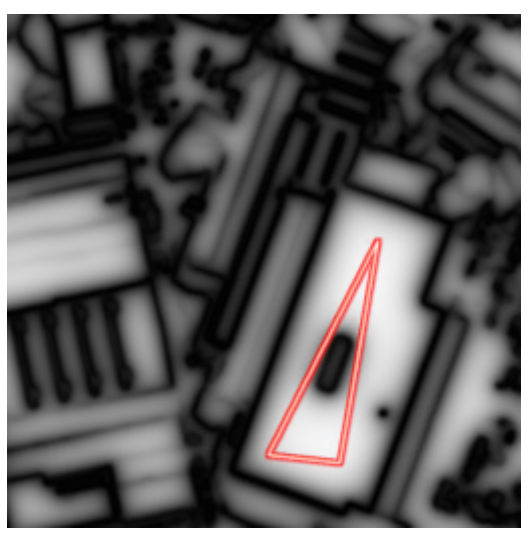

(c) initial edge map, $\epsilon=0.05$ and initial configuration

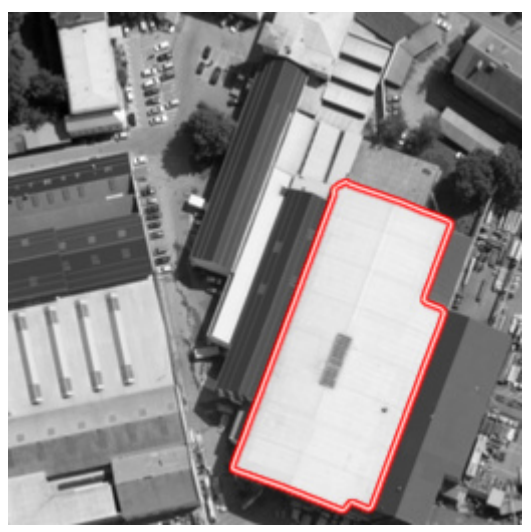

(e) final result, $\epsilon=0.001, \eta=2$

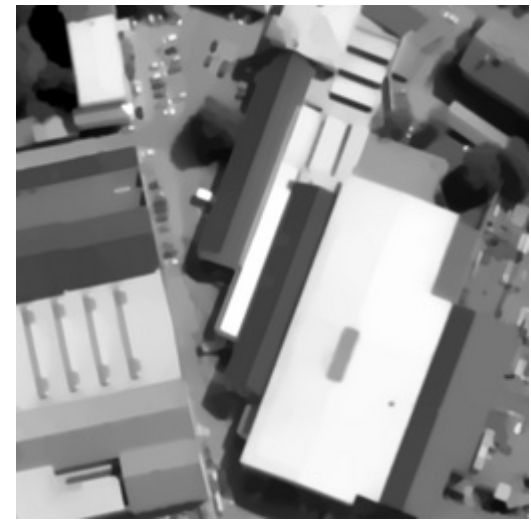

(b) applying TV- $L^{1}$ ISS filtering

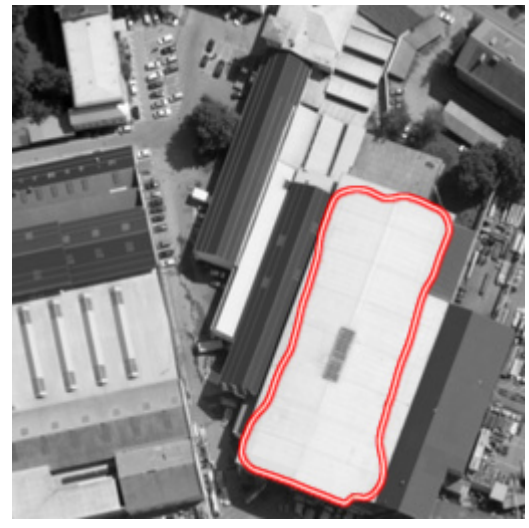

(d) intermediate stage of evolution

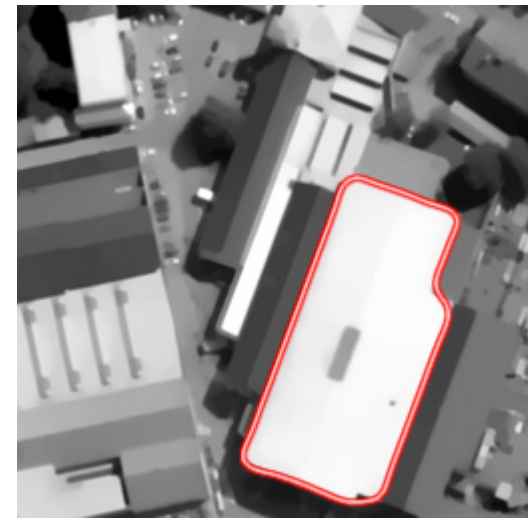

(f) dropping the curvature weight

FIG. 4.2. In this example, a piecewise constant Mumford-Shah energy combined with the external force induced by the phase field function 4.4 and the weighted Willmore functional has been minimized (cf. equation 2.16). 


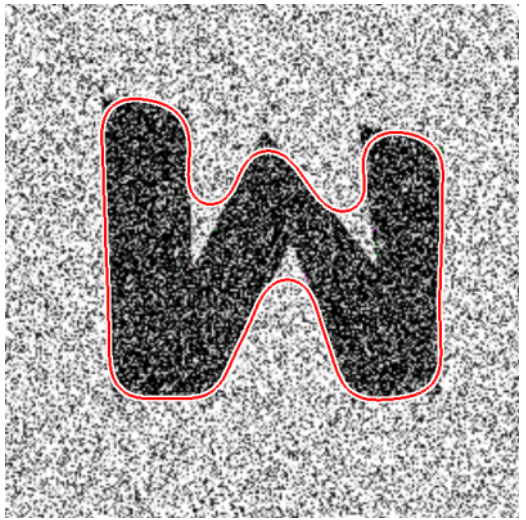

(a) Steady state for $\eta=\infty$.

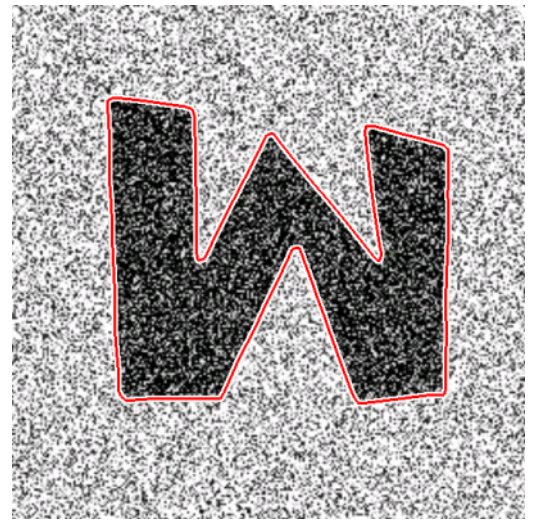

(b) Steady state for $\eta=\frac{1}{4}$.

FIG. 4.3. The image on the left shows the steady state of 4.4 on a noisy image using Willmore regularization, which yields oversmoothing especially at sharp angles. The image on the right demonstrates the advantage of using the weighted Willmore energy on polygonal shapes. The same weight for the fidelity term $\left(\gamma_{\mathrm{MS}}=10^{7}\right)$ has been used for both computations.

interactive segmentation process starts by computing the evolution of the relaxed inverse scale space equation (4.2) until the main features of the desired object are clearly visible. Then we have chosen an initial $\epsilon=0.1$ (the image domain is scaled to have a unit edge length) and compute the minimum of the energy 4.3 (see Figure 4.1(b). At that stage we are mainly interested in driving the contour towards an attracting regime that is close enough to the correct boundaries of the object, such that the scale can safely be refined without risking the computation of a false local minimum.

In this phase we keep the contour smooth until it hits interesting features and start evolving according to 2.10 by chosing a large sensitivity value of $\eta,(=25$ in our test cases, see (2.7) for the role of $\eta$ ). Once we have reduced $\epsilon$ and reached a fine scale, in which corners become significant, we reduce $\eta$ to a value around 1 . To demonstrate that the formation of corners is truly due to the curvature dependent weight and not only due the multiscale generation of the external potential, we have increased $\eta$ at the final segmentation shown in Figure 4.1(e) and recomputed the new stationary point. We observe that the contour flips back to an overly smooth approximation of the boundary as shown in Figure 4.1(f)

All the proposed formulations lead to entirely intrinsic evolution equations. The condition of the discrete approximation of the differential operators depends on the spacing of the grid points on the curve. Even though the projection helps significantly to reduce the effect of tangential shifts, the implicit time stepping and the movement of the curve into concavities often leads to an irregular spacing, which we correct by a retriangulation. Furthermore, it is desirable to maintain a sufficient number of discrete grid points close to the corners to be able to approximate the geometry well. In the one-dimensional case, retriangulation can be easily achieved using the arclength parametrization. For two-dimensional manifolds a simple volume-preserving mesh regularization technique (see Sec. 4.2 ) can be used.

In Figure 4.2 we have experimented with a variational segmentation model on a similar input image. We have minimized the piecewise-constant Mumford-Shah model 
(cf. 2.16), combined with the weighted Willmore functional:

$$
E\left[c_{i}, \Gamma\right]=\int_{\Gamma} G(h) \mathrm{d} A+\gamma_{\mathrm{MS}} \sum_{i=1,2} \int_{\Omega_{i}}\left(c_{i}-u_{0}\right)^{2} \mathrm{dx}+\gamma_{\mathrm{AT}} \int_{\Gamma} w_{\epsilon} \mathrm{d} A,
$$

where $w_{\epsilon}$ is the phase-field function from the solution of (4.3). In this example we chose $\gamma_{\mathrm{MS}}=10^{8}$ and $\gamma_{\mathrm{AT}}=10^{6}$, so that the piecewise-constant Mumford-Shah term is the dominant external force contribution.

In order to compute the gradient of the energy functional numerically, integrals over $\Omega_{1}$ and $\Omega_{2}$, i.e., the regions divided by $\Gamma$ have to be evaluated. We used an eikonal solver to compute a signed distance function starting with initial signed distances on the corners of all cells that are intersected by $\Gamma$. These cells are tesselated into triangles to achieve subpixel accuracy along the interface.

In the experiments, the region-based partitioning has proven quite insensitive to strong misleading gradients in the image (see the small hole in the roof). This would be difficult to achieve with a local feature based external force term only, as for example in (1.1).

In 4.2(f) we again verify the positive impact of the curvature dependent weighting term. Also, the beneficial effect of the inverse scale space method is apparent: already at a short time the regularized image reflects the main contour of the building very sharply. The irrelevant smaller objects, which could have a negative influence on the segmentation, are not yet visible.

Figure 4.3 demonstrates the benefit of using the weighed Willmore regularization on polygonal shapes: undesirable oversmoothing at corners can be prevented without sacrificing an overall regularizating effect, which is responsible for avoiding high-frequency perturbations of the curve due to noisy input data. In this computation, we have not applied any denoising on the input image.

An indicative test case is the segmentation of Kanizsa's triangle (Figure 4.1.3), which aims at the detection of subjective contours [36, i.e., the identification of an object which is not perceptible in its raw form, but only through continuation of partially visible boundaries. The human observer can easily identify the triangle as the most plausible simple geometric object, that fits into the given geometric configuration. Due to the sharp concavities, the object is a good candidate for feature preserving regularization. To segment the interior triangle we proceeded as follows. Since the input image Fig. 4.4(a) is clean and does not contain fine-scale patterns, we did not need to apply any pre-filtering. To obtain an initial edge map in Fig. 4.4(b), we chose the phase-field parameter as $\epsilon_{0}=0.2$ for Eq. 2.10). To help the circle shrink towards an attracting regime, corresponding to the corners of the triangle, we have added a modest length penalization to compute the first stationary solution $\Gamma_{0}$ with $\eta_{0}$ set to 100, which basically turns off the feature-preservation (see Fig. 4.4(c)p. The curve $\Gamma_{0}$ is now sufficiently close enough to allow to switch off the length penalization, which would only unnecessarily smooth the curve and undo the corner-preservation regularization. We set $\epsilon_{1}=0.05$ and increased the curvature sensitivity by setting $\eta_{1}$ to 10 which computes the solution shown in Fig. 4.4(d). The steep external force field, already pulls the snake very close to the desired triangle shape. The curvaturedependency is however not strong enough to avoid the regularization on the tip of the corners. After reducing $\eta_{2}$ further to 1 , we finally arrive at the segmentation result shown in Figs. 4.4(e) and 4.4(f).

4.2. Surface smoothing. One of the most natural applications of feature-preserving regularization techniques is feature-preserving smoothing. Furthermore, there 


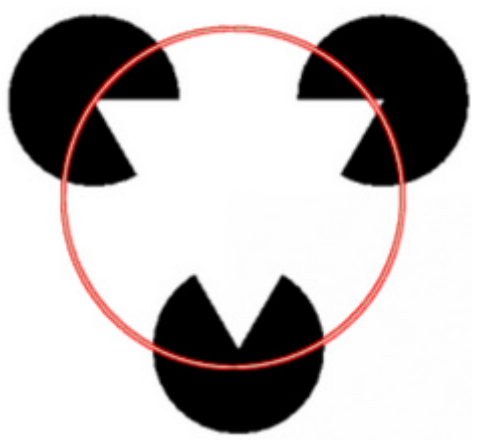

(a) initial configuration

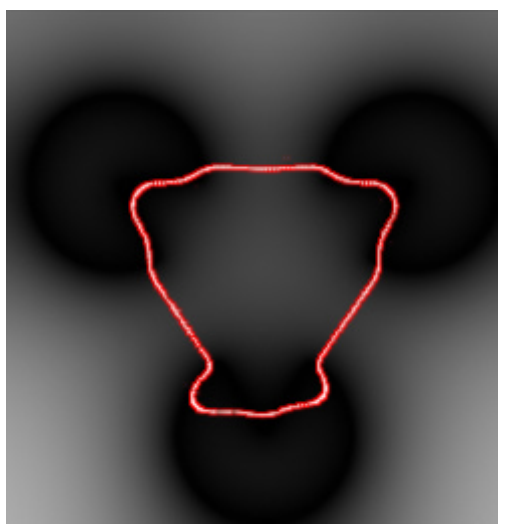

(c) intermediate step of the segmentation

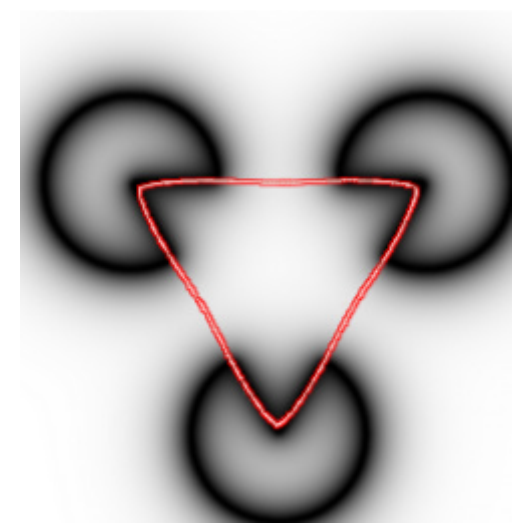

(e) final result on top of the finest edge map used

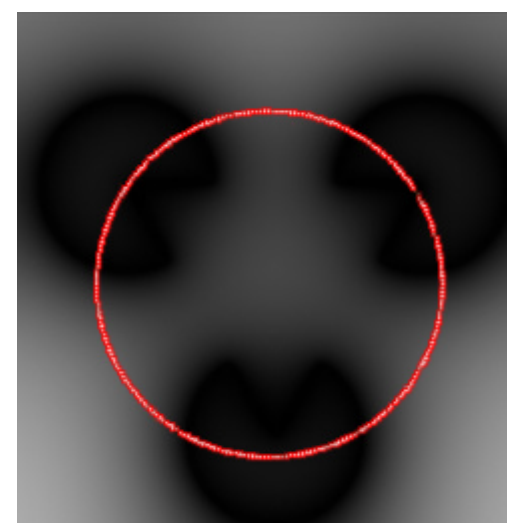

(b) initial edge map

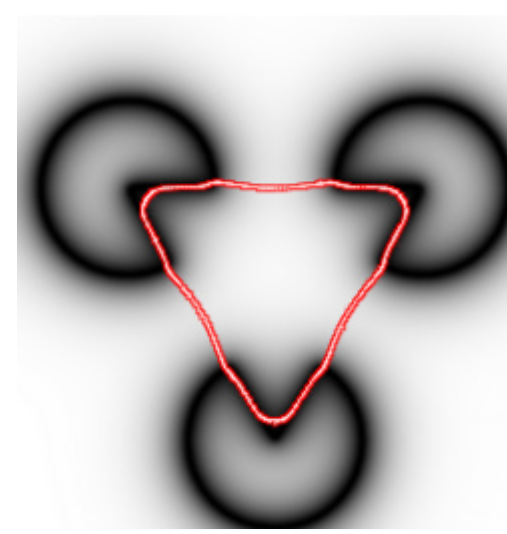

(d) intermediate step of the segmentation for a finer edge map

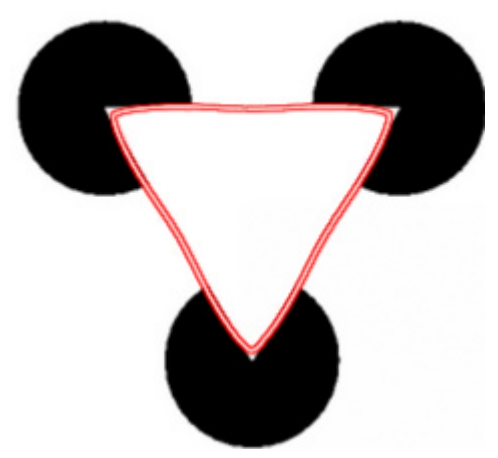

(f) final result on top of the original image

FIG. 4.4. Psycho-visual segmentation: segmentation of the Kanizsa triangle. 


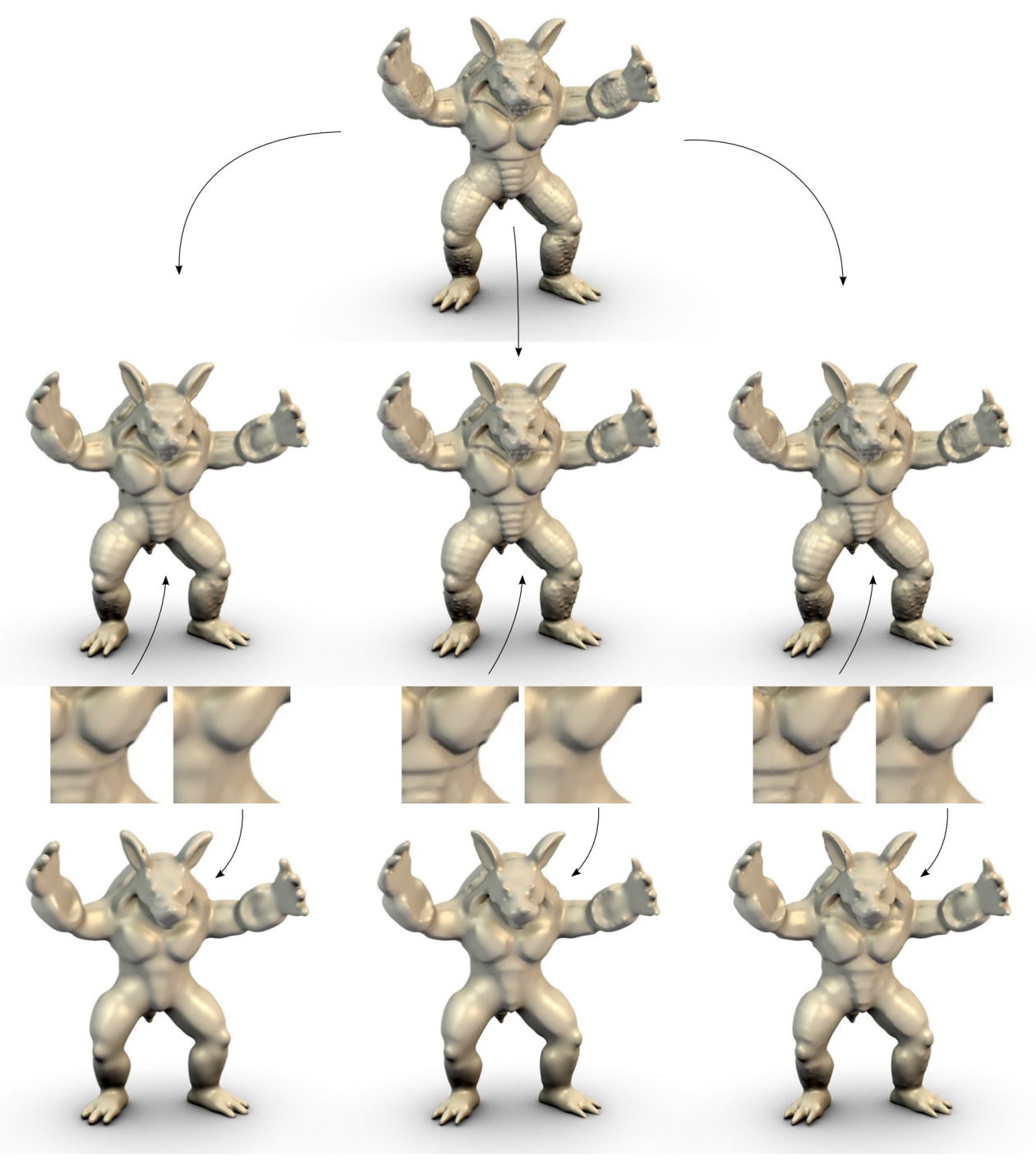

FIG. 4.5. Different multi-scales on the armadillo data set. The left column shows the evolution of the original surface (top) under standard Willmore flow at time steps 1 and 4. In the middle row, the simplified surface diffusion 2.7 has been computed. The right column shows the weighted Willmore flow. Time steps 1,3 and 5 are depicted. The timestep $\tau$ is $10^{-8}$ and $\eta=10$.

exists a wide range of problems, that are based on scale-space techniques. We have considered two basic approaches:

\subsubsection{Generating a multiscale by computation of initial value prob-}

lems. Similarly to PDE-based scale-space approaches in image processing, we can simply evolve the initial input geometry $\Gamma_{0}$ under the evolution equation 2.7 to obtain a family of smoothed surfaces $\Gamma(t)$.

A related approach is to consider the gradient-flow of the weighted Willmore 


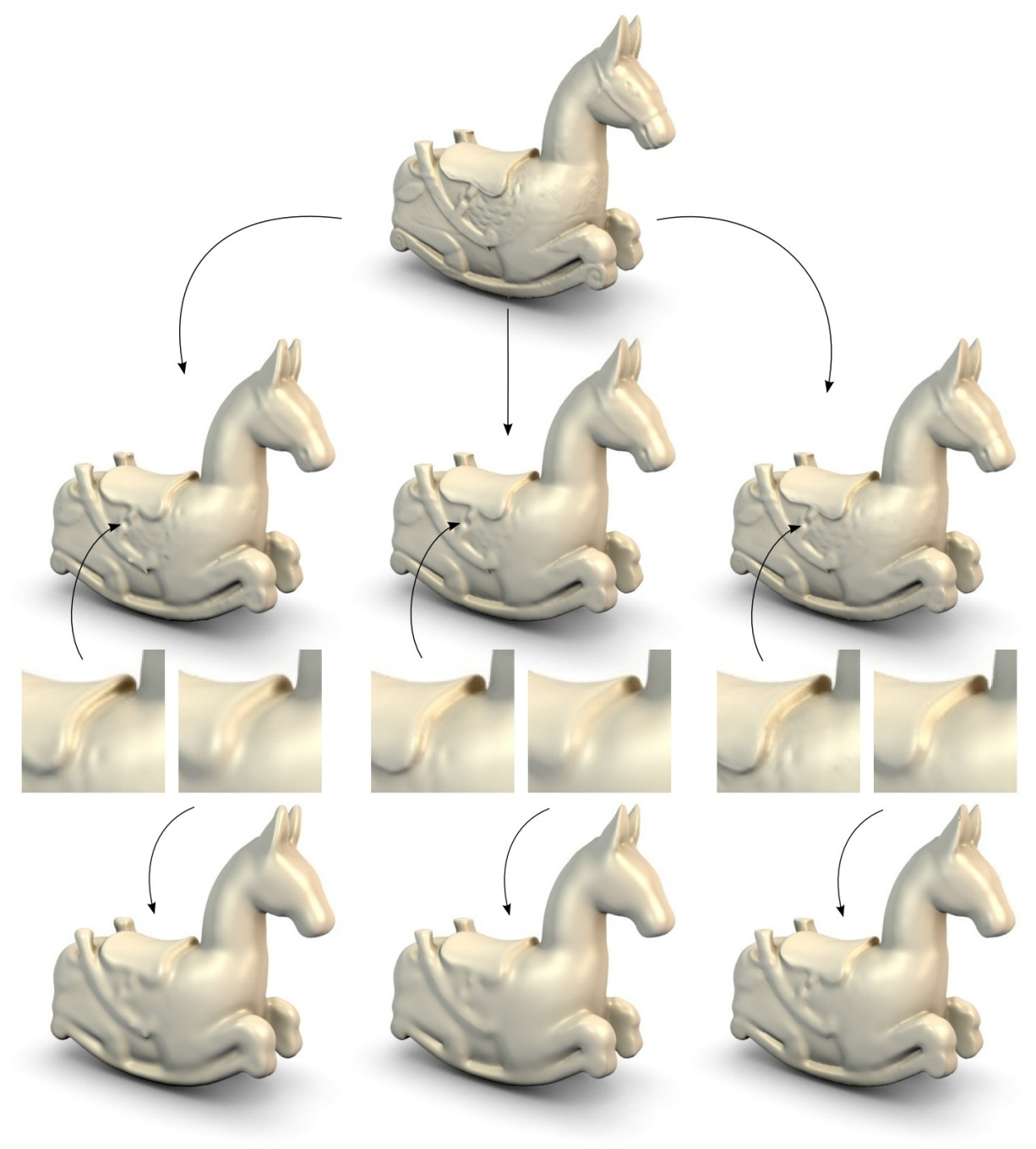

FIG. 4.6. Comparison of different higher-order multi-scales on the horse data set. The left column shows the evolution of the original surface (top) under standard Willmore flow at time steps 1 and 4. In the middle row, the simplified surface diffusion 2.7) has been computed. The right column shows the weighted Willmore flow. The timestep $\tau$ is $10^{-9}$ and $\eta=10$. All variants remove geometric texture very rapidly, but the curvature-dependent flows also preserve important features.

functional

$$
\left.x_{t}=-\operatorname{grad}_{L^{2}(\Gamma(t))} W[\Gamma(t))\right] \quad \text { with } \Gamma(0)=\Gamma_{0} .
$$

Both versions define a scale-space operator $S(T): \Gamma_{0} \mapsto \Gamma(T)$. By construction we have $S(0)=\mathbb{1}$ and $S\left(T_{1}+T_{2}\right)=S\left(T_{2}\right) \circ S\left(T_{1}\right)$ (semigroup property) a fundamental requirement for the construction of scale-spaces. In Figures 4.5 and 4.6 we show a comparison of standard Willmore flow (left column) and the two curvature-dependent evolutions, i.e., (2.7) (middle column) and weighted Willmore flow (right column). While standard Willmore flow rounds-off all parts of the surface homogeneously, the 

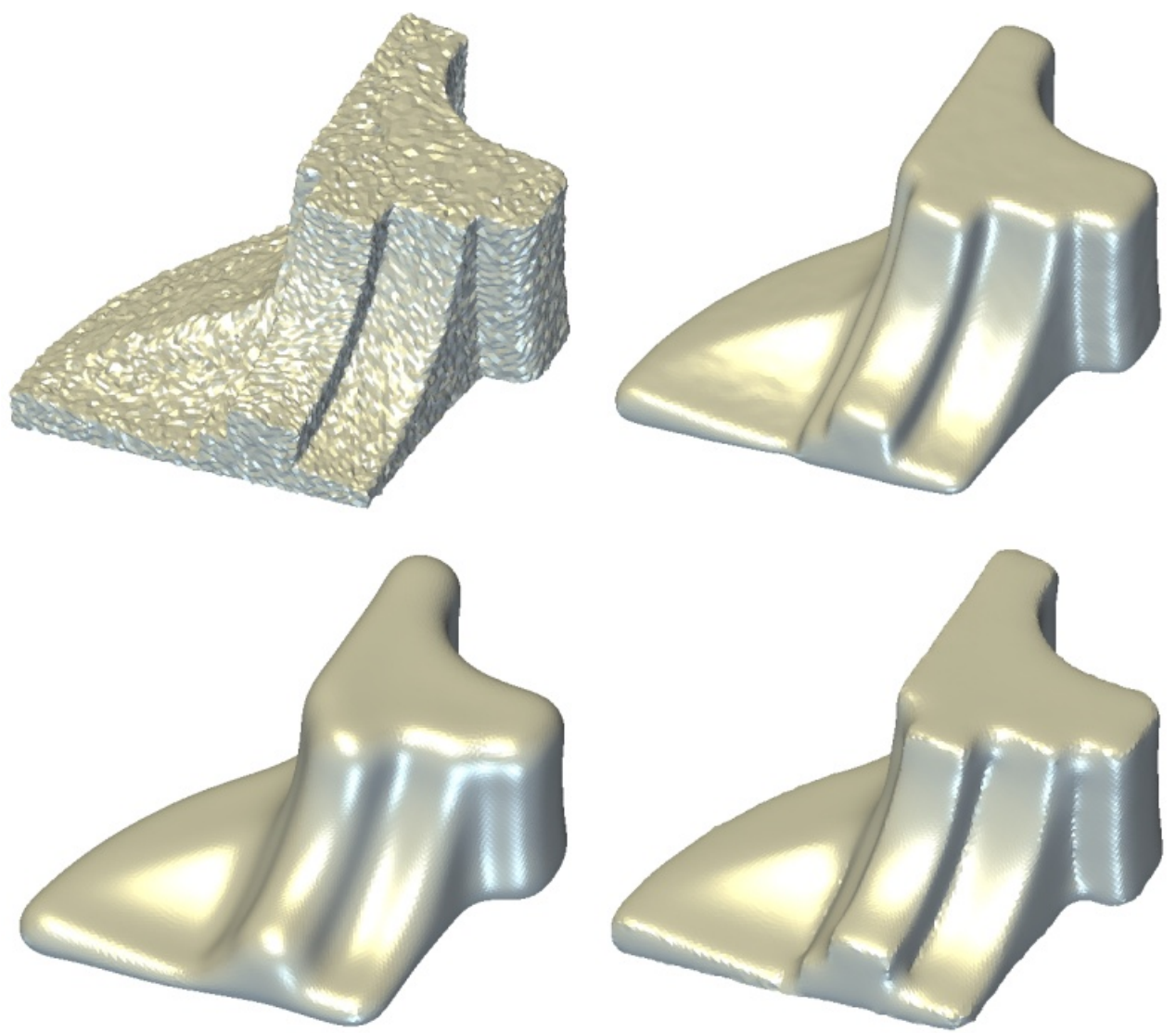

FIG. 4.7. Denoising of the fandisk data set with fidelity. Top left shows the noisy model. On the top right the surface is denoised with standard mean curvature flow. The bottom left shows denoising with Willmore flow. The bottom right shows the denoising result with the weighted Willmore flow.

weighted versions produce a much more appealing coarse to fine scale, in which important geometric features such as smooth creases and tips are preserved significantly better. The curvature sensitivity parameter has been set to $\eta=10$ in both variants.

The horse mesh contains a fine-level geometric texture. During Willmore flow, this texture is removed already in the early stage of the evolution, however, the creases are unnaturally rounded off. During the evolution of the weighted Willmore-flow, the texture is removed quickly as well, but the shape of the object on a macro-scale is well preserved.

4.2.2. Energy minimization with a fidelity term. Inspired by the set-up of the Rudin-Osher-Fatemi model, we might also consider the variational problem:

$$
\min _{\mathcal{A}} \underbrace{\int_{\Gamma} G(h) \mathrm{d} A}_{=W[\Gamma]}+\lambda \int_{\Gamma} d\left(\cdot, \Gamma_{0}\right) \mathrm{d} A
$$

for a given, initial noisy surface $\Gamma_{0}$. In contrast to the Euclidean case, the choice of the fidelity function $d: \mathbb{R}^{d+1} \times \mathbb{R}^{d+1} \rightarrow \mathbb{R}_{0}^{+}$is not straightforward in the case of 
surfaces. Naturally the Hausdorff distance would be a good candidate for measuring the fidelity of $\Gamma$ to the initial surface, we chose $d\left(x, \Gamma_{0}\right):=\operatorname{dist}\left(x, \Gamma_{0}\right)^{2}$ which simplifies the minimization process, because $d$ can be precomputed for a given initial surface. In this model, $\lambda$ defines a balance between the regularity of $\Gamma$ and how close the smoothed surface should be to the original surface. For fixed $\lambda$, the overall functional is minimized.

Even with spatial acceleration structures, such as KD-tree or bounding volume hierarchies, the on-the-fly computation of the fidelity integral would be costly, because the projection of a point onto a triangulated surface involves at least a local search. Instead, we computed the distance function of $\Gamma_{0}$ on the bounding box on a uniform grid, in a two-step procedure:

1. Initialize the distance function in the vicinity of $\Gamma_{0}$. More precisely, for all triangles $T$ of the discrete noisy surface $\Gamma_{0, h}$, find all cubes $C_{T, i}$, that are intersected by $T$, compute for all nodes of $C_{T, i}$ the distance to $T$. If this distance is smaller than a potentially previously computed value, update its value.

2. Now that the values of the distance function are known close to the interface, we can use a solver for the eikonal equation $\|\nabla u\|=1$ to extend the distance function onto the whole domain (i.e. the enlarged bounding box of $\Gamma_{0}$ ).

In Figure 4.7 we show the results of this approach applied to the well-known fandisk dataset. We have compared our functional with the area functional and the Willmore energy and adjusted $\eta$ in each case to obtain visually appealing results to have a somewhat fair comparison. Not surprisingly the curvature-dependent approach preserves the creases much better than the other two. In this computation we have set $\eta=5$.

Note that the construction of the signed distance function also introduces some numerical errors. The result can thus not be compared with pure mesh-based smoothing techniques. We also observe that creases do not evolve to perfectly sharp feature lines, an effect which hints to an analogy to the Euclidean case, in which LCIS overcomes staircaising effects.

4.2.3. Remarks. An inconvenient side-effect of the movement of vertices during the evolution is that the mesh may degenerate, leading to a very high condition number. Hence, depending on the number of steps taken and the initial regularity of the mesh, retriangulation of the mesh can become unavoidable. We have found the scheme proposed by Bänsch et al. 4] very useful, since it is fast, easy to implement and preserves the enclosed volume of the mesh, which is particularly for higher-order flows a desirable property.

Compared to the range of existing feature-preserving surface denoising techniques in the literature, for instance the anisotropic geometric diffusion techniques by Clarenz et al. 21], the subdivision surface discretization Bajaj and $\mathrm{Xu}$ [3], the discrete exterior calculus formulation of Hildebrandt and Polthier 32] or bilateral filtering of Jones et al. 35, we focused on the different qualitative smoothing which has a "rounding" effect, in contrast to the "shrinking" effect that is typical for second order techniques, that are based on the penalization of area. The iterative feature preserving smoothing of normal fields Tasdizen \& Whitaker [54] and fitting for surfaces represented by level set functions has a similar effect, although it is strictly speaking not a fourth order flow. The variational setting is flexible and easily extends to other applications and variational set-ups, for instance to apply recent developments for cartoon-texture decomposition methods of images onto surfaces.

Our finite element computations have used the OpenMesh library for the traversal 
of elements [8].

5. Summary and conclusion. Motivated by low-curvature image simplifiers in image processing, we have presented geometric and fully intrinsic fourth-order feature preserving regularization techniques by drawing analogies to surface diffusion in a weighted $H^{-1}$ metric and a weighted Willmore functional. We observed, that a simplified form of the first variant has very similar qualitative properties, but is much easier to implement and can be computed much more efficiently. Due to the need for a suitable energy functional for feature-preserving regularization of geometric variational problems, we extended the finite element formulation of Rusu to a more general mean-curvature dependent energy functional. We applied the new regularization methods to segmentation of aerial images and were able to precisely extract object boundaries with sharp corners. We also compared the different variants in the context of surface denoising and again verified very similar results, which allows to choose the model depending on the problem context. When a flow equation is sufficient, the simplified variant of surface diffusion is more convenient, since it is easier to implement than weighted Willmore flow. Both methods smooth out geometric texture quickly without destroying sharp features.

From our first promising results, we see a large potential to improve the regularization in a wide range of geometric optimization problems. We can expect further improvement by extending the isotropic curvature weight by an anisotropic one in the spirit of Clarenz et al. [21] or Diewald [26].

The recently presented numerical scheme by Dziuk 29 for parametric Willmore flow completely cancels tangential shifts. The derivation of an analogous weighted formulation would be an interesting extension to circumvent the mesh regularization steps (and their associated numerical errors) we currently employ to avoid degenerated elements.

Acknowledgments. We thank Jean-Michel Morel for very helpful discussions and Guy Gilboa for very useful conversations on inverse scale space techniques that were applied in this paper. The horse model is provided courtesy of INRIA and IMATI, the armadillo model is taken from the Stanford 3D Scanning Repository. We also thank Aerowest GmbH and Vexcel Corporation for providing the aerial images (Figures 4.1 and 4.2. The fandisk model is courtesy H. Hoppe. This work has been supported by the NSF grants AST-0244498, ACI-0321917, the ONR grants N000140410078 and N000140810363 and the Department of Defense.

\section{REFERENCES}

[1] L. Ambrosio and V. M. Tortorelli, On the approximation of free discontinuity problems, Boll. Un. Mat. Ital. B, 6 (1992), pp. 105-123.

[2] G. Aubert and L. Blanc-Féraud, Some remarks on the equivalence between $2 D$ and 3D classical snakes and geodesic active contours, International Journal of Computer Vision, 34 (1999), pp. 19-28.

[3] C. L. BAJAJ AND G. Xu, Anisotropic diffusion of subdivision surfaces and functions on surfaces, ACM Transactions on Graphics (TOG), 22 (2003), pp. 4-32.

[4] E. Bänsch, P. Morin, And R.H. Nochetto, Finite element methods for surface diffusion: the parametric case, Journal of Computational Physics, 203 (2005), pp. 321-343.

[5] G. Bellettini and M. Paolini, Anisotropic motion by mean curvature in the context of finsler geomety, QUADERNO, 49 (1994).

[6] A.L. Bertozzi And J.B. Greer, Low curvature image simplifiers: global regularity of smooth solutions and laplacian limiting schemes, Comm. Pure Appl. Math., 57 (2004), pp. 764790 . 
[7] A. Bertozzi, J. Greer, S. Osher, And K. Vixie, Nonlinear regularizations of TV based PDEs for image processing, in AMS Series of Contemporary Mathematics, George Gasper Gui-Qiang Chen and Joseph Jerome, eds., vol. 371, 2005, pp. 29-40.

[8] M. Botsch, S. Steinberg, S. Bischoff, and L. Kobbelt, Openmesh - a generic and efficient polygon mesh data structure, in OpenSG Symposium, 2002.

[9] B. Bourdin ANd A. Chambolle, Implementation of a Finite-Element approximation of the Mumford-Shah functional, Numer. Math., 85 (2000), pp. 609-646.

[10] M. Burger, A framework for the construction of level set methods for shape optimization and reconstruction, Interfaces and Free Boundaries, 5 (2003), pp. 301-329.

[11] M. BuRger, Numerical simulation of anisotropic surface diffusion with curvature-dependent energy, J. Comp. Phys., 203 (2005), pp. 602-625.

[12] M. Burger, G. Gilboa, S. Osher, and X. Jinjun, Noninear inverse scale space methods, Tech. Report 05-66, University of California, Los Angeles, 2005.

[13] V. Caselles, F. Catté, T. Coll, and F. Dibos, A geometric model for active contours in image processing, Numer. Math., 66 (1993), pp. 1-31.

[14] V. Caselles And B. Coll, Snakes in movement, SIAM J. Numer. Analy., 33 (1996), pp. 24452456.

[15] V. Caselles, R. Kimmel, and G. Sapiro, Geodesic active contours, International Journal of Computer Vision, 22 (1997), pp. 61-79.

[16] T. Chan And L. VeSe, An active contour model without edges, in Scale-Space Theories in Computer Vision. Second International Conference, Scale-Space '99, Corfu, Greece, September 1999, M. Nielsen, P. Johansen, O. F. Olsen, and J. Weickert, eds., Lecture Notes in Computer Science; 1682, Springer, 1999, pp. 141-151.

[17] T. F. Chan, J. Shen, and L. Vese, Variational PDE models in image processing, in Notes of the AMS, vol. 50(1), 2003, pp. 14-26.

[18] Tony F. Chan and Luminita A. Vese, Active contour and segmentation models using geometric PDEs for medical imaging, in Malladi, R. (Ed.), Geometric Methods in biomedical Image Processing, Series: Mathematics and Visualization, Springer, 2002, pp. 63-75.

[19] U. Clarenz, The wulff-shape minimizes an anisotropic willmore functional, Interfaces and Free Boundaries, 6 (2004), pp. 351-359.

[20] U. Clarenz, U. Diewald, G. Dziuk, M. Rumpf, and R. Rusu, A finite element method for surface restoration with smooth boundary conditions, Computer Aided Geometric Design, 21 (2004), pp. 427-445.

[21] U. Clarenz, U. Diewald, and M. Rumpf, Nonlinear anisotropic diffusion in surface processing, in Proceedings Visualization 2000, B. Hamann T. Ertl and A. Varshney, eds., 2000, pp. 397-405.

[22] U. Clarenz, G. Dziuk, and M. Rumpf, On generalized mean curvature flow in surface processing, in Geometric analysis and nonlinear partial differential equations, H. Karcher and S. Hildebrandt, eds., Springer, 2003, pp. 217-248.

[23] T. Cootes And C. TAYlor, Active shape models- - smart snakes, 1992.

[24] K. Deckelnick and G. Dziuk, Discrete anisotropic curvature flow of graphs, Mathematical Modelling and Numerical Analysis, 33 No 6 (1999), pp. 1203-1222.

[25] K. Deckelnick, G. Dziuk, and C. M. Elliott, Fully discrete simi-implicit second order splitting for anisotropic surface diffusion of graphs, Tech. Report 33, Universität Magdeburg, 2003.

[26] U. Diewald, Anisotrope Krümmungsflüsse parametrischer Flächen sowie deren Anwendung in der Flächenverarbeitung, $\mathrm{PhD}$ thesis, University of Duisburg-Essen, 2005.

[27] M. P. Do CARmo, Riemannian Geometry, Birkhäuser, Boston, 1992.

[28] M. Droske and M. Rumpf, A level set formulation for Willmore flow, Interfaces and Free Boundaries, 6 (2004), pp. 361-378.

[29] G. Dzıuk, Computational parametric Willmore flow, Tech. Report 13-07, Fakultät für Mathematik und Physik, University of Freiburg, 2007.

[30] M. Elsey and S. Esedoglu, Analogue of the total variation denoising model in the context of geometry processing, Tech. Report 07-31, UCLA, 2007.

[31] S. Esedoglu and S.J. Osher, Decomposition of images by the anisotropic Rudin-OsherFatemi model, Comm. Pure Appl. Math., 57 (2004), pp. 1609-1626.

[32] K. Hildebrandt and K. Polthier, Anisotropic filtering of non-linear surface features, in Computer Graphics Forum, vol. 23(3), 2004.

[33] M. Hintermüller AND W. RING, A second order shape optimization approach for image segmentation, SIAM Appl. Math, 64 (2003), pp. 442-467.

[34] K. Ito, K. Kunisch, AND L. Zhilin, Level-set function approach to an inverse interface problem, Inverse Problems, 17 (2001), pp. 1225-1242. 
[35] T. Jones, F. Durand, And M. Desbrun, Non-iterative, feature-preserving mesh smoothing, in Computer Graphics (SIGGRAPH 2003 Proceedings), 2003, pp. 943-949.

[36] G.K. Kanizsa, Subjective contours, in Scientific American, vol. 234, 1976.

[37] M. Kass, A. Witkin, And D. Terzopoulos, Snakes: Active contour models, International Journal of Computer Vision, 1 (1988), pp. 321-331.

[38] Satyanad Kichenassamy, Arun Kumar, Peter J. Olver, Allen Tannenbaum, and AnTHONY J. YEZZI, Gradient flows and geometric active contour models, in ICCV, 1995, pp. 810-815.

[39] R. Kimmel and A.M. Bruckstein, Regularized Laplacian zero crossings as optimal edge integrators, International Journal of Computer Vision, 53 (2003), pp. 225-243.

[40] E. Kuwert And R. Schätzle, Gradient flow for the Willmore functional, Comm. Anal. Geom., 10 (2002), pp. 1228-1245 (electronic).

[41] U.F. Mayer and G. Simonett, A numerical scheme for axisymmetric solutions of curvature driven free boundary problems with applications to the Willmore flow, Interphases and Free Boundaries, 4 (2002), pp. 1-22.

[42] U. F. Mayer And G. Simonett, Self-intersections for the Willmore flow, in Evolution Equations: Applications to Physics, Industry, Life Sciences and Economics. EVEQ 2000 Conference in Levico Terme (Trento, Italy), Birkhäuser, Basel, 2003, pp. 341-348.

[43] D. Mumford And J. Shah, Optimal approximation by piecewise smooth functions and associated variational problems, Comm. Pure Appl. Math., 42 (1989), pp. 577-685.

[44] M. Nitzberg, D. Mumford, and T. Shiota, Filtering, Segmentation and Depth (Lecture Notes in Computer Science Vol. 662), Springer-Verlag Berlin Heidelberg, 1993.

[45] P. Perona And J. Malik, Scale space and edge detection using anisotropic diffusion, in IEEE Computer Society Workshop on Computer Vision, 1987.

[46] K. Polthier, Computational aspects of discrete minimal surfaces, in Global Theory of Minimal Surfaces, Proc. of the Clay Mathematics Institute Summer School, D. Hoffman, ed., CMI/AMS, 2005.

[47] L. Rudin And E. Osher, S. AD FAtemi, Nonlinear total variation based noise-removal, Physica D, 60 (1992), pp. 259-268.

[48] R. Rusu, An algorithm for the elastic flow of surfaces, Preprint Mathematische Fakultät Freiburg, 01-35 (2001), p. .

[49] G. SAPIRO, Vector (self) snakes: a geometric framework for color, texture and multi-scale image segmentation, in Proc. IEEE Int. Conf. Image Proc. (ICIP), Lausanne, 1996.

[50] — Geometric Partial Differential Equations and Image Analysis, Cambridge University Press, 2001.

[51] O. Scherzer, M. Grasmair, H. Grossauer, M. Haltmeier, and F. Lenzen, Variational methods in imaging, Springer, 2009.

[52] L. Simon, Existence of Surfaces Minimizing the Willmore Functional, Commun. Analysis Geom., 1(2) (1993), pp. 281-326.

[53] G. Simonetr, The Willmore Flow near spheres, Diff. and Integral Eq., 14(8) (2001), pp. 10051014.

[54] T. Tasdizen, R. Whitaker, P. Burchard, and S.J. Osher, Geometric surface processing via normal maps, ACM Transactions on Graphics, 22 (2003), pp. 1012-1033.

[55] V. Thомée, Galerkin - Finite Element Methods for Parabolic Problems, Springer, 1984.

[56] J. Tumblin and G. TuRK, A boundary hierarchy for detail-preserving contrast reduction, in Proceedings of the SIGGRAPH 1999 annual conference on Computer Graphics, August 8-13, 1999, Los Angeles, CA, USA, 1999, pp. 83-90.

[57] C.-F. Westin, L. M. Lorigo, O. D. Faugeras, W. E. L. Grimson, S. Dawson, A. Norbash, AND R. KIKINIS, Segmentation by adaptive geodesic active contours, in Proceedings of MICCAI 2000, Third International Conference on Medical Image Computing and ComputerAssisted Intervention, A. M. DiGioia and S. Delp, eds., Pittsburgh, October 11-14 2000, pp. 266-275.

[58] T.J. Willmore, Riemannian Geometry, Claredon Press, Oxford, 1993.

[59] G. WulfF, Zur Frage der Geschwindigkeit des Wachsthums und der Auflösung der Kristallflächen, Zeitschrift der Kristallographie, 34 (1901), pp. 449-530. 
Appendix. As an instructive alternative to the Finite Element discretization, we present straightforward spatial finite difference approximations of the geometric differential operators that are needed for the numerical solution of (2.7). For a discrete approximation of a Jordan-curve $\Gamma$ by a closed polygon with $n$ vertices $x_{i}$, $i=0, \ldots, n-1$, we define

$$
l_{i+\frac{1}{2}}:=\left\|x_{i+1}-x_{i}\right\| \quad \text { and } \quad l_{i}:=\frac{l_{i+\frac{1}{2}}+l_{i-\frac{1}{2}}}{2}
$$

using the convention $x_{i}=x_{i} \bmod n$. Then the piecewise constant difference quotient approximations of $\nabla_{\Gamma_{h}} u$ for $u: \Gamma_{h} \rightarrow \mathbb{R}$ on the segment $\left[x_{i}, x_{i+1}\right)$ is given by

$$
\nabla_{h, \Gamma_{h}} u_{i+\frac{1}{2}}:=\frac{u_{i+1}-u_{i}}{l_{i+\frac{1}{2}}}
$$

Furthermore we approximate the divergence at $x_{i}$ for a piecewise constant function $v$ (corresponding to a one-dimensional vectorfield) on the polygon segments by

$$
\operatorname{div}_{h, \Gamma_{h}} v_{i}:=\frac{v_{i+\frac{1}{2}}-v_{i-\frac{1}{2}}}{l_{i}} .
$$

The discrete approximation of the discrete Laplace-Beltrami operator $\Delta_{\Gamma_{h}}$ is then given by

$$
\Delta_{\Gamma_{h}} u_{i}:=\operatorname{div}_{h, \Gamma_{h}} \nabla_{h, \Gamma_{h}} u_{i}=\frac{\frac{u_{i+1}-u_{i}}{l_{i+\frac{1}{2}}}-\frac{u_{i}-u_{i-1}}{l_{i-\frac{1}{2}}}}{l_{i}} .
$$

After incorporating the mobility $g$, we obtain

$$
\operatorname{div}_{h, \Gamma_{h}}\left(g \nabla_{h, \Gamma_{h}}\right) u_{i}=\frac{g_{i+\frac{1}{2}} \frac{u_{i+1}-u_{i}}{l_{i+\frac{1}{2}}}-g_{i-\frac{1}{2}} \frac{u_{i}-u_{i-1}}{l_{i-\frac{1}{2}}}}{l_{i}}
$$

by evaluating $g$ on the midpoints of the intervals. This approximation corresponds to the Finite Element approximation using lumped masses and a barycentre quadrature rule. The relation (5.1) for all vertices $x_{i}$ can be expressed by a matrix $\mathbf{L}[g]$ and hence 2.7 is discretized in space by

$$
\bar{X}_{t}+\mathbf{L}[g] \mathbf{L}[1] \bar{X}=0, \quad \bar{X}(0)=\bar{X}_{0},
$$

for every spatial component of $\Gamma$, where again $\bar{X}$ denote the vector of coefficients. Note, that $\mathbf{L}[g]$ is also changing in time, due to the dependence on $l$.

Even though the scalar part in 2.13 could be discretized similarly, it is not straightforward to devise a semi-implicit time-stepping scheme for weighted Willmore flow. This is much easier with a Finite Element approach using the weak formulation from Thm. 2.5.

In higher dimensions the corresponding analogues of the Finite Element approximations lead to discrete exterior calculus (cf. [46] for details). 